\title{
Modeling Jupiter's magnetosphere: Influence of the internal sources
}

\author{
E. Chané, ${ }^{1,2}$ J. Saur, ${ }^{1}$ and S. Poedts ${ }^{3}$ \\ Received 4 December 2012; revised 29 March 2013; accepted 1 April 2013; published 22 May 2013.
}

[1] We introduce a new model to study Jupiter's magnetosphere and how it interacts with the solar wind. We first derive a set of one-fluid MHD equations to consistently include the ion-neutral collisions in Jupiter's ionosphere and the mass loading in the Io torus. The mass loading and the subsequent radial mass transport in Jupiter's magnetosphere leads to a deviation from full corotation of the magnetospheric plasma. Ion-neutral collisions in Jupiter's ionosphere and subsequent transport of angular momentum out into the magnetosphere acts to spin up the magnetosphere's plasma. Our model explicitly includes mass loading in the Io plasma torus and an inner boundary region, which represents the effects of Jupiter's ionosphere. We present the results of five model runs where different mass loading rates and ionospheric conductances are used. For these model runs, we consider an antiparallel interplanetary magnetic field and a strong solar wind dynamic pressure, resulting in a compressed magnetosphere. The results are compared with analytical models, in situ measurements, and remote-sensing observations. Our azimuthal velocity profiles and the position of the corotation breakdown are in quantitative agreement with theoretical predictions by Hill $(1979,2001)$ and Saur et al., (2004a), and Voyager observations. The total current flowing into and out of the ionosphere is $48.7 \mathrm{MA}$, which is in agreement with estimates from measurements and analytical models. Using the field aligned electric current $j_{\|}$to determine the position of the aurorae, we find that our main auroral oval is associated, as expected, with the position of the corotation breakdown (between $20.6 \mathrm{R}_{\mathrm{J}}$ and $30.1 \mathrm{R}_{\mathrm{J}}$ for the different model runs). The discontinuity in the main oval observed by Radioti et al. (2008) is also present in our results, where it is caused by an asymmetry in the pressure distribution, due to the interaction between the rotating plasma and the magnetopause.

Citation: Chané, E., J. Saur, and S. Poedts (2013), Modeling Jupiter's magnetosphere: Influence of the internal sources, J. Geophys. Res. Space Physics, 118, 2157-2172, doi:10.1002/jgra.50258.

\section{Introduction}

[2] The Jovian magnetosphere is shaped by the interaction of the solar wind with the planet's magnetic field, but it is also strongly influenced by the massive internal plasma source Io combined with the fast rotation of the planet. Due to the latter two effects, the coupling of the magnetosphere to Jupiter's atmosphere and ionosphere (also called MI coupling) is very different compared to the Earth. The full nature of this coupling, how it responds to changing internal and external conditions, and how these changing conditions

\footnotetext{
${ }^{1}$ Institut für Geophysik und Meteorologie, Universität zu Köln, Cologne, Germany.

${ }^{2}$ Space Science Center, University of New Hampshire, Durham, New Hampshire, USA.

${ }^{3}$ Center for Mathematical Plasma Astrophysics, KU Leuven, Leuven, Belgium

Corresponding author: E. Chané, Institut für Geophysik und Meteorologie, Universität zu Köln, Albertus-Magnus-Platz, 50923 Cologne, Germany. (chane@geo.uni-koeln.de)

(C)2013. American Geophysical Union. All Rights Reserved. 2169-9380/13/10.1002/jgra.50258
}

affect the auroral activity, however, still remains unclear in many aspects.

[3] The Jovian magnetosphere's dominant internal plasma source, Io, which orbits Jupiter at a radial distance of $5.9 \mathrm{R}_{\mathrm{J}}$, is the most volcanically active body in our solar system. This volcanism is the root cause for Io's sulfur dioxide atmosphere, from which about metric ton of mass per second is lost into the Jovian magnetosphere. The neutral matter from Io accumulates primarily along Io's orbit where it is eventually ionized to form the Io plasma torus [see reviews by Thomas et al., 2004; Khurana et al., 2004; Krupp et al., 2004; Saur et al., 2004b].

[4] The heavy plasma injected into the magnetosphere is transported radially outward, most likely due to a centrifugal instability [Ioannidis and Brice, 1971; Hill, 1976; Vasyliunas, 1983]. Due to the centrifugal force, the heavy magnetospheric plasma is mostly confined to the magnetic equator in the plasma sheet. As a result of conservation of angular momentum, the outward moving plasma will subcorotate in the absence of any other torques acting on it, i.e., the plasma will rotate with an angular velocity smaller than the angular velocity of Jupiter. The subcorotating plasma modifies the magnetic field and generates Lorentz forces 
$(\mathbf{j} \times \mathbf{B}$, where $\mathbf{j}$ and $\mathbf{B}$ represent the current density and the magnetic field, respectively), which act to accelerate the plasma toward corotation. The angular momentum needed to sustain the constant reacceleration of plasma comes from Jupiter and its atmosphere. Elastic collisions of neutral particles in Jupiter's atmosphere with the plasma in Jupiter's ionosphere provide momentum to the plasma and are the root cause for the acceleration of the magnetospheric plasma. At a certain radial distance, the coupling of the atmosphere/ionosphere to the magnetosphere (MI coupling) cannot sustain the acceleration of the magnetospheric plasma anymore and corotation breaks down [see, e.g., Hill, 1979; Huang and Hill, 1989; Pontius, 1997; Bunce and Cowley, 2001]. This occurs at radial distances in the range between 15 to $30 \mathrm{R}_{\mathrm{J}}$. Inside of $15 \mathrm{R}_{\mathrm{J}}$, the magnetosphere is nearly fully corotating due to the described coupling to Jupiter's atmosphere/ionosphere. According to Hill [1979], the location of the breakdown is controlled by the total mass injection rate into the magnetosphere, the planetary magnetic field strength, and the conductance of the planetary ionosphere.

[5] The region where the breakdown of corotation occurs coincides with the maximum field aligned electric current with direction away from Jupiter. These field lines map into the main auroral oval of Jupiter as first suggested by Hill [2001] and Cowley and Bunce [2001]. At radial distances a few $R_{J}$ above Jupiter's ionosphere, the charge carrier density is extremely low, and thus, electrons need to be accelerated to large velocities to maintain the fieldaligned electric current density. These accelerated electrons precipitate into the atmosphere and generate the aurora. An illustration of this scenario is given in Figure 1. In addition, it was shown by Ray et al. [2009, 2010, 2012] that including the field-aligned potentials in the corotation enforcing current system affects the field-aligned current density, the electron precipitation, the Pedersen conductivity, and the transfer of angular momentum to the magnetosphere. It means that, due to saturation (or partial saturation) of the ionosphere-magnetosphere coupling currents, the corotation breaks down closer to Jupiter due to the field-aligned potentials.

[6] We developed a model which includes a fast rotating magnetized central body surrounded by an ionospheric region - where ion-neutral collisions are present-and a toroidal region where mass loading is applied. With this model, we perform 3-D global MHD numerical experiments, and the values are chosen as close as possible to resemble the interaction between the solar wind and Jupiter's magnetosphere. In particular, the influence of the Io torus mass loading rate and of the ionospheric conductance are studied in the present paper. Several analytical models, which include the Io mass loading rate and the ionospheric conductance, already exist, e.g., Hill [1979, 2001], Cowley and Bunce [2001], Cowley et al. [2002, 2003], and Nichols and Cowley [2003]. These models demonstrate how the conductance and mass loading rate affect the azimuthal velocity and the magnetosphere-ionospheric coupling currents in Jupiter's magnetosphere. While Hill [1979, 2001] assume a dipole field in the Jovian magnetosphere, Cowley and Bunce [2001] and Cowley et al. [2002, 2003] considered an empirical magnetic field which describes the deformation of the planetary magnetic field due to the azimuthal

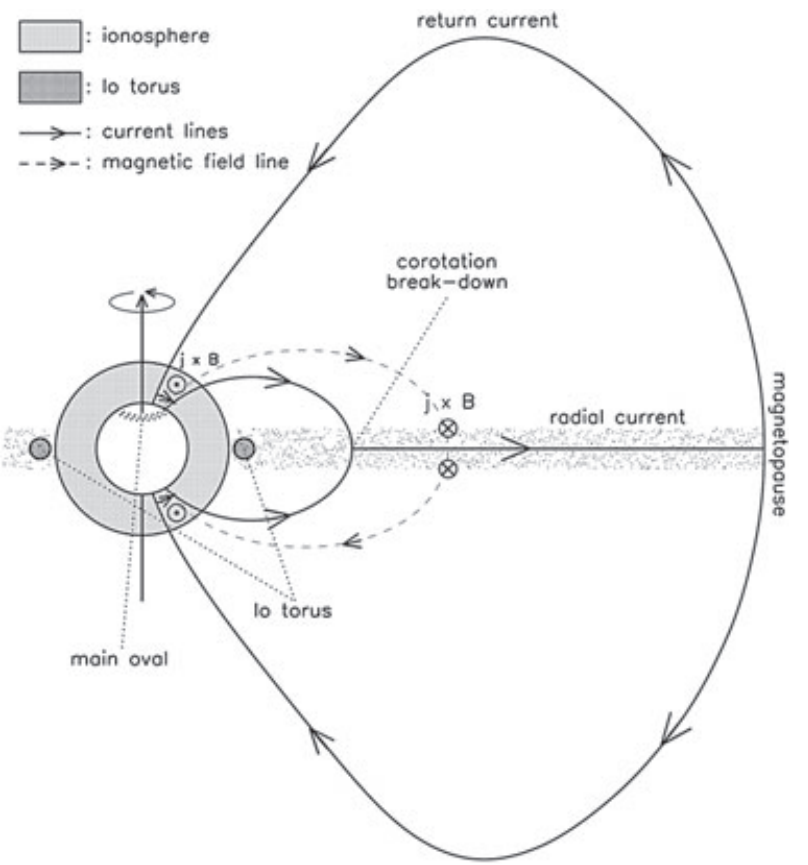

Figure 1. Sketch of the Jovian dayside magnetosphere and of the corotation enforcing currents. It displays in light gray the ionosphere (where the ion-neutral collisions take place), the Io torus in dark gray (where plasma is created through ionizations of Iogenic neutrals) and the current sheet plasma (represented by the small dots). The black solid lines represent the current, while the dashed line shows the magnetic field. The magnetic Lorentz force $(\mathbf{j} \times \mathbf{B})$ is shown in the current sheet (where it points in the direction of corotation) and in the ionosphere (where it acts against corotation). In this sketch, the return currents are assumed to be located at the magnetopause.

currents. Nichols and Cowley [2003] present results for a wide range of values for the ionospheric conductance and the mass loading rate applying both the dipole field model of Hill [1979, 2001] and the current sheet magnetic field model of Cowley and Bunce [2001] and Cowley et al. [2002, 2003].

[7] The present study differs in several aspects from these analytical models. First of all, while the analytic models are naturally not self-consistent in the set of plasma parameters; in particular, the magnetic field topology is prescribed in these models, and the effect of the solar wind on the plasma parameters is not taken into account, our magnetic field is consistently computed. In addition, our model is threedimensional, while the analytic models are axisymmetric and cannot account for the local time asymmetries present in Jupiter's magnetosphere. The advantage of the analytic models is that they are not affected, unlike ours, by numerical diffusion introduced by limited mesh resolution (typical for global MHD computations). The analytic models lead to general descriptions of the plasma angular velocity and currents as a function of basic input parameters, while we can only present the results of a few model runs (five in the present paper).

[8] Only a few global MHD models of the Jovian magnetosphere-solar wind interaction are published in the literature. In these models, the inner boundary is always 
located far from the planetary surface: $8 \mathrm{R}_{\mathrm{J}}$ for the most recent publications [Moriguchi et al., 2008], but sometimes as far as $30 \mathrm{R}_{\mathrm{J}}$ [Ogino et al., 1998]. As a result, the Io torus is never introduced explicitly in the numerical domain, and the mass loading is just treated as a boundary condition.

[9] The first global MHD numerical models of the Jovian magnetosphere were independently and nearly simultaneously performed by Miyoshi and Kusano [1997] and Ogino et al. [1998]. In Miyoshi and Kusano [1997], the inner boundary was located at $30 \mathrm{R}_{\mathrm{J}}$, where the angular velocity was fixed to $50 \%$ of the Jovian angular velocity. Their model showed how Jupiter's fast rotation generates dawn-dusk asymmetries.

[10] Ogino et al. [1998] mainly studied how the fast rotation of Jupiter influences the shape of the magnetosphere. Their model was later used to study the influence of the solar wind dynamic pressure and magnetic field on the magnetosphere [Walker et al., 2001], the current systems in the magnetosphere [Walker and Ogino, 2003], and the ejection of plasmoids in the tail [Fukazawa et al., 2005]. In all these publications, the inner boundary is located at $21 \mathrm{R}_{\mathrm{J}}$, the mass loading is not introduced in the equations but occurs through the inner boundary, and no ionosphere model is present.

[11] Finally, Moriguchi et al. [2008] presented a new model where the influence of the ionosphere is treated as a boundary condition (note that Hansen et al. [2000] and Jia et al. [2012] use a similar approach in their model of Saturn's magnetosphere): the field-aligned currents at the inner boundary $\left(8 R_{J}\right)$ are mapped along the magnetic field lines down to $1 R_{J}$, where a potential equation is solved on a two-dimensional shell, assuming prescribed and uniform Hall and Pedersen conductances. The ionospheric potential is then mapped back to $8 \mathrm{R}_{\mathrm{J}}$ where it is used as an inner boundary condition to specify the velocity components perpendicular to the magnetic field $\mathbf{v}_{\perp}$. The magnetic field boundary condition at $8 \mathrm{R}_{\mathrm{J}}$ is such that the radial component of the magnetic field is set to the internal dipole field, i.e., the radial component of the perturbation field is set to zero. In the article, the authors focused on the analysis of the current systems and on the ejection of plasmoids. Since their inner boundary was located at $8 \mathrm{R}_{\mathrm{J}}$, the Io torus could not be included in their model runs.

[12] In our numerical model, we use a different approach; we implement an extended ionospheric region inside the numerical domain (between 4.5 and $8.5 \mathrm{R}_{\mathrm{J}}$ ) and explicitly include the mass loading in the Io plasma torus, which due to numerical reason is located at $10 \mathrm{R}_{\mathrm{J}}$. As a result, we cannot model the inner magnetosphere of Jupiter realistically but include key physical parameters (mass loading and ionospheric conductance) to provide valid results farther from Jupiter. This is because at large distances from the ionosphere and from the mass loading region, the dominantly controlling parameters which determine magnetospheric properties such as plasma density, velocity, and magnetic field are the ionospheric conductance and the total mass loading, whereas the size of the ionosphere and the position where the mass loading occurs only weakly influence those magnetospheric properties. Our model is explained in detail in the next section, where we also derive the required equations and explain the assumptions, simplifications, and boundary conditions. Then, in section 3 , the results of our model runs are presented: After the general model results are shown in section 3.1 , we show in section 3.3 the modeled locations of the corotation breakdown and the azimuthal velocity profiles, which we compare with theoretical model predictions and Voyager measurements. To our knowledge, our model is the first numerical MHD model which quantitatively compares the azimuthal velocity profile with theory and observations. In section 3.4, we study the aurorae and magnetosphere-ionosphere coupling in our model and how they compare with remote-sensing observations and in situ measurements. Our conclusions are presented in section 4.

\section{Model}

\subsection{Model Overview}

[13] Our model takes a different approach compared to previous numerical models for Jupiter's and Saturn's magnetosphere [e.g., Ogino et al., 1998; Hansen et al., 2000; Walker and Ogino, 2003; Moriguchi et al., 2008; Jia et al., 2012]. It explicitly includes an extended ionospheric region between 4.5 and $8.5 \mathrm{R}_{\mathrm{J}}$ (Figure 1). In the ionospheric region, the MHD equations are explicitly solved where we include ion-neutral collisions to account for the momentum transfer from Jupiter's atmosphere to the plasma of Jupiter's ionosphere. Our MHD boundary conditions are set at the inner boundary, i.e., below the ionospheric region. The choice of the location and extension of the ionospheric region is for numerical reasons. An inner boundary closer than $4.5 \mathrm{R}_{\mathrm{J}}$ would increase the maximal magnetic field strength inside the numerical domain and thus also the Alfvén velocity at the inner boundary leading to small time steps and unrealistic computational times. Note that the position of the ionosphere - as long as it is clearly within the position of corotation breakdown (i.e., $R_{p} \ll L_{0}$ )—does not or only very weakly affect the azimuthal velocity profile or the position of corotation breakdown. According to Hill [1979], the L-shell of the corotation breakdown is: $L_{0}=\sqrt[4]{2 \pi \Sigma R_{p}^{2} B_{p}^{2} / \dot{M}}$, where $R_{p}$ is the planetary radius, $B_{p}$ is the planetary surface dipole magnetic field strength, and $\dot{M}$ is the total rate of production and outward transport of plasma mass. However, if we consider the position of the corotation breakdown $\left(R_{0}\right)$ in meters rather than in units of $R_{p}$, and as a function of the planetary magnetic moment $M_{p}$, then we find: $R_{0}=\sqrt[4]{2 \pi \Sigma\left(\mu_{0} / 4 \pi M_{p}\right)^{2} / \dot{M}}$, with $M_{p}=4 \pi / \mu_{0} B_{p} R_{p}^{3}$ and $\mu_{0}$ is the permeability of free space. One can see that this distance $R_{0}$ does not depend on the position of the ionosphere. The same holds for the subcorotation in the Io plasma torus, which only depends on the ionospheric and the magnetospheric conductances [Saur et al., 2004a, equation (12)].

[14] The real extension of the ionosphere in our model needs to be grossly enhanced to numerically resolve the ionospheric region. In our model, we explicitly include a plasma torus where we control the mass loading rate. The torus is centered around $10 \mathrm{R}_{\mathrm{J}}$ to be sufficiently far away from the ionospheric region of our model and from the region of corotation breakdown (Figure 1). With this approach, we do not model a realistic ionosphere in several respects, but the inclusion of the ion-neutral collision frequency allows us to control the ionospheric conductances, which are key parameters of the magnetosphere-ionosphere coupling [Hill, 1979, 2001; Cowley and Bunce, 2001]. 
Therefore, we call this inner model region "ionospheric region" and not ionosphere. Although the inner magnetosphere is not realistic in our model, the magnetospheric areas far away from the ionospheric region and from the Io torus are modeled realistically and display properties in accordance with analytically derived expectations calculated with our ionospheric conductances and mass loading rates. The main goal of our model is to describe Jupiter's magnetosphere in the regions where corotation breaks down and farther outside as correctly as possible.

[15] Our approach has main advantages compared to models previously applied to Jupiter's or Saturn's magnetosphere [e.g., Ogino et al., 1998; Hansen et al., 2000; Walker and Ogino, 2003; Moriguchi et al., 2008]. In these models, an inner boundary at radial distances far above the real ionosphere is assumed, e.g., $8 \mathrm{R}_{\mathrm{J}}$ in the model of Moriguchi et al. [2008]. Several models simply fix the plasma velocity and the magnetic field at their inner boundary above the ionosphere and thus do not include a coupling to an ionosphere with finite resistivity [e.g., Ogino et al., 1998; Hansen et al., 2000; Walker and Ogino, 2003; Fukazawa et al., 2005]. Models which include the effect of the ionosphere map the electric current from the inner boundary to an infinitely thin ionosphere situated at $1 \mathrm{R}_{\mathrm{J}}$. The ionospheric electric field is calculated with prescribed ionospheric conductances and is then mapped out again to the inner boundary to provide the boundary condition for the plasma velocity. However, in these models, the magnetic field boundary conditions are generally applied at their inner boundary, i.e., above the ionosphere, which severely constrain the magnetosphereionosphere coupling. Moriguchi et al. [2008], for example, set the radial component of the perturbation magnetic field $\mathbf{B}_{1}$ to zero at $8 \mathrm{R}_{\mathrm{J}}$. The perturbation magnetic field is the part of the magnetic field, which deviates from the internal magnetic field of Jupiter. This boundary condition has severe consequences. As shown in Appendix B, setting the radial component of the perturbation field to zero $\left(\delta B_{r}=0\right)$ on a sphere with radius $R$ implies that, at a given latitude, the azimuthal electric current density $j_{\phi}$ averaged over all longitudes vanishes (i.e., $\left\langle j_{\phi}\right\rangle=0$ ). At the radial distances where the boundary conditions are set, the electric current is nearly field aligned. Therefore, the magnetic field will have both positive and negative $B_{\phi}$ along a given latitude. While this is not necessarily an issue for the Earth's magnetosphere, this is problematic in a magnetosphere shaped by its own rotation like the Jovian magnetosphere, since such magnetic field orientations correspond to, both, subcorotation and supercorotation along all longitudes at a certain radial distance. This might be the reason why Moriguchi et al. [2008] and other models obtain longitudes with strong supercorotation in regions of the magnetosphere where subcorotation is expected and observed. The advantage of our model is that we set our boundary conditions for all MHD fields below the ionospheric region at $4.5 \mathrm{R}_{\mathrm{J}}$ and fully solve the MHD equations within the ionospheric region and the magnetosphere. With this approach, we omit the magnetic field boundary constraints on top of the ionosphere, which affect Jupiter's magnetosphere-ionosphere coupling. In section 3.3, we quantitatively compare our results with theoretical predictions and demonstrate that our model approach realistically matches results with respect to magnetospheric subcorotation.

\subsection{Model Derivation}

[16] The ion-neutral collisions taking place in the ionosphere of Jupiter and the mass loading located in the Io torus are essential to understand and to model the Jovian magnetosphere, specially with respect to the current systems associated with the main auroral oval. We thus include these two phenomena in our model. This is done by means of source terms in the one-fluid MHD equations. How to correctly and consistently describe these source terms in the framework of one-fluid MHD requires some attention. We therefore start the derivation with separate sets of fluid equations for the electrons and ions, where the source terms pertaining to mass loading and collisions have been derived from first principles from the Boltzmann equation [see, e.g., Schunk, 1975]. Since we aim to derive a single-fluid model in the present paper, we only consider here one ion species which we assume to be singly charged. As a basic set of equations for the electrons and for the ions, we use the continuity, the momentum, and the energy (thermal and kinetic) equations as follows:

$$
\begin{aligned}
\frac{\partial \rho_{s}}{\partial t}= & -\nabla \cdot\left(\rho_{s} \mathbf{v}_{\mathbf{s}}\right)+\kappa_{s} \\
\frac{\partial}{\partial t}\left(\rho_{s} \mathbf{v}_{\mathbf{s}}\right)+\nabla \cdot\left(\rho_{s} \mathbf{v}_{\mathbf{s}} \mathbf{v}_{\mathbf{s}}\right)= & -\nabla p_{s} \\
& \pm n_{s} e\left(\mathbf{E}+\mathbf{v}_{\mathbf{s}} \times \mathbf{B}\right)+\rho_{s} v_{s n}\left(\mathbf{v}_{\mathbf{n}}-\mathbf{v}_{\mathbf{s}}\right) \\
& +\rho_{s} \mathbf{g}+\kappa_{s} \mathbf{v}_{\mathbf{n}} \\
\frac{\partial}{\partial t}\left(\frac{3}{2} n_{s} k T_{s}+\frac{1}{2} \rho_{s} v_{s}^{2}\right)= & -\nabla \cdot\left(\frac{1}{2} \rho_{s} v_{s}^{2} \mathbf{v}_{\mathbf{s}}\right) \\
& -\nabla \cdot\left(\frac{3}{2} n_{s} k T_{s} \mathbf{v}_{\mathbf{s}}\right)-\nabla \cdot\left(p_{s} \mathbf{v}_{\mathbf{s}}\right) \\
& \pm n_{s} e\left(\mathbf{E}+\mathbf{v}_{\mathbf{s}} \times \mathbf{B}\right) \cdot \mathbf{v}_{\mathbf{s}} \\
& +\frac{n_{s} m_{s} v_{s n}}{m_{s}+m_{n}}\left(3 k\left(T_{n}-T_{s}\right)+m_{n}\left(\mathbf{v}_{\mathbf{s}}-\mathbf{v}_{\mathbf{n}}\right)^{2}\right) \\
& +\rho_{s} v_{s n}\left(\mathbf{v}_{\mathbf{n}}-\mathbf{v}_{\mathbf{s}}\right) \cdot \mathbf{v}_{\mathbf{s}}+\rho_{s} \mathbf{v}_{\mathbf{s}} \cdot \mathbf{g} \\
& +\kappa_{s}\left(\frac{1}{2} v_{n}^{2}+\frac{3}{2} \frac{T_{n} k}{m_{s}}\right)
\end{aligned}
$$

The index $s$ stands for species, i.e., for ions or electrons. These three equations describe the time evolution of the mass density $\left(\rho_{s}\right)$, the momentum $\left(\rho_{s} \mathbf{v}_{\mathbf{s}}\right)$, and the thermal and kinetic energies $\left(\frac{3}{2} n_{s} k T_{s}+\frac{1}{2} \rho_{s} v_{s}^{2}\right)$ in the inertial frame. They display the two-fluids equations along with source terms which include the following: (1) the ion-neutral collisions, (2) the mass loading, and (3) the Jovian gravity. These source terms are explained in detail below. In these equations, $p_{s}$ represents the thermal pressure, $n_{s}$ is the number density, $e$ is the elementary charge, $\mathbf{E}$ is the electric field, $\mathbf{B}$ is the magnetic field, $\mathbf{g}$ is the gravitational acceleration of Jupiter, $k$ is the Boltzmann constant, and $m_{s}$ is the mass of species $s$. In addition, $\mathbf{v}_{\mathbf{n}}, m_{n}$, and $T_{n}$ are the bulk velocity, the mass, and the temperature of the neutral particles, respectively. The mass density production rate associated with the Io torus is noted $\kappa$, and $v_{s n}$ is the collision frequency with the neutral particles in Jupiter's ionosphere.

[17] In equation (1), the term $\kappa_{s}$ describes the mass density production rate caused by ionization in the Io torus. In equation (2), the term $\rho_{s} v_{s n}\left(\mathbf{v}_{\mathbf{n}}-\mathbf{v}_{\mathbf{s}}\right)$ describes how the plasma is accelerated or decelerated by the neutral gas of the Jovian 
atmosphere (depending on its velocity with respect to $\mathbf{v}_{\mathbf{s}}$ ) because of the collisions. The derivation of this term can be found, for instance in Schunk [1975]. In this equation, the gravitational force exerted by Jupiter is also present $\left(\rho_{s} \mathbf{g}\right)$, with $\mathbf{g}=\frac{G M_{J}}{r^{3}} \mathbf{r}$, where $G$ is the gravitational constant and $M_{J}$ is the mass of Jupiter. Note that as explained in details in section 2.3 , in our model runs an artificially enhanced $G M_{J}$ is used for practical and numerical reasons (55 times higher than the normal value). Since gravity decreases rapidly with radial distance, this enhanced gravity only has a minor effect on the model output. In addition, the momentum of the neutrals that were ionized in the Io torus $\left(\kappa_{S} \mathbf{v}_{\mathbf{n}}\right)$ is added to the plasma.

[18] In equation (3), the temperature coupling between the plasma and the neutral particles $\left(\frac{n_{s} m_{s} v_{s n}}{m_{s}+m_{n}} 3 k\left(T_{n}-T_{s}\right)\right)$ (sometimes called collisional energy transfer in the literature), and the Joule heating $\left(\frac{n_{s} m_{s} v_{s n}}{m_{s}+m_{n}} m_{n}\left(\mathbf{v}_{\mathbf{s}}-\mathbf{v}_{\mathbf{n}}\right)^{2}\right)$, both caused by the collisions with the neutral gas in the ionosphere, are included (for the derivation, see Schunk [1975]). While in this equation, the temperature coupling and the Joule heating actually show how the temperature of the plasma is affected by collisions with neutrals, their kinetic energy is affected by the neutral drag $\left(\rho_{s} v_{s n}\left(\mathbf{v}_{\mathbf{n}}-\mathbf{v}_{\mathbf{s}}\right) \cdot \mathbf{v}_{\mathbf{s}}\right)$. The neutral drag shows that, when the neutral particles of the atmosphere flow in the same direction as the plasma but are faster, the kinetic energy of the plasma is increased by the collisions. Otherwise, it decreases. The term $\rho_{s} \mathbf{v}_{\mathbf{s}} \cdot \mathbf{g}$ represents the change in the kinetic energy of the plasma caused by the gravity field of the planet. Finally, according to energy conservation, we add the kinetic and thermal energy of the neutral particles that were ionized $\left(\kappa_{s} \frac{1}{2} v_{n}^{2}\right.$ and $\kappa_{s} \frac{3}{2} \frac{T_{n} k}{m_{s}}$, respectively).

[19] To derive our set of one-fluid MHD equations, we assume that the plasma consists of electrons and one kind of ion. Combining equations (1), (2), and (3) with Ampère's law and Faraday's law of induction, we derive a complex set of one-fluid MHD equations for the mass density, the momentum density, the magnetic field, and the total energy density which includes more than 50 terms. We then perform a scale analysis to determine which of these terms can be neglected without altering the important physical processes of the model. In order to estimate the importance of each term, we calculate their typical timescales (Appendix A) for the two regions where source terms were introduced, i.e., the ionosphere and the Io torus. The timescales are evaluated based on typical values for all the variables present in our equations for these two regions. The terms related to very long timescales are then considered to be negligible and can be removed from the equations. For example, using this method for the momentum equation, we find that the timescale associated with $\nabla \cdot(\rho \mathbf{v v})$ is nine orders of magnitude smaller than the timescale associated with $\nabla \cdot\left(\frac{m_{e}}{n e^{2}} \mathbf{j} \mathbf{j}\right)$ in the ionosphere and 16 orders of magnitude smaller in the Io torus (Table A3). As a result, we have neglected the later term in the equations. After applying these simplifications, we find the following set of one-fluid MHD equations:

$$
\frac{\partial \rho}{\partial t}=-\nabla \cdot(\rho \mathbf{v})+\kappa
$$

$$
\begin{gathered}
\frac{\partial}{\partial t}(\rho \mathbf{v})=-\nabla \cdot\left(\rho \mathbf{v} \mathbf{v}+\underline{\underline{\mathbf{I}}}\left(p+\frac{B^{2}}{2 \mu_{0}}\right)-\frac{\mathbf{B B}}{\mu_{0}}\right) \\
+\rho \mathbf{g}+\rho v_{\text {in }}\left(\mathbf{v}_{\mathbf{n}}^{\text {iono }}-\mathbf{v}\right)+\kappa \mathbf{v}_{\mathbf{n}}^{\text {torus }}, \\
\frac{\partial \mathbf{B}}{\partial t}=\nabla \times(\mathbf{v} \times \mathbf{B})-\nabla \times\left(\frac{\mathbf{j} \times \mathbf{B}}{n e}\right), \\
\frac{\partial U}{\partial t}=-\nabla \cdot\left((U+p) \mathbf{v}+\frac{B^{2}}{2 \mu_{0}} \mathbf{v}-\mathbf{v} \cdot \frac{\mathbf{B B}}{\mu_{0}}\right) \\
+\rho v_{\text {in }}\left(\mathbf{v}_{\mathbf{n}}^{\text {iono }}-\mathbf{v}\right)\left(\frac{\mathbf{v}_{\mathbf{n}}^{\text {iono }}}{\chi^{\text {iono }}+1}+\frac{\chi^{\text {iono }} \mathbf{v}}{\chi^{\text {iono }}+1}\right) \\
+\left(\rho \frac{p_{n}^{\text {iono }}}{\rho_{n}^{\text {iono }}}-p\right) \frac{3}{1+\chi^{\text {iono }}} v_{i n}+\frac{\mathbf{B}}{\mu_{0}} \cdot \nabla \times\left(-\frac{\mathbf{j} \times \mathbf{B}}{n e}\right) \\
+\frac{\kappa\left(v_{n}^{\text {torus }}\right)^{2}}{2}+\frac{3 \kappa p_{n}^{\text {torus }}}{\chi^{\text {torus }} \rho_{n}^{\text {torus }}}+\rho \mathbf{v} \cdot \mathbf{g},
\end{gathered}
$$

where $\mathbf{I}$ is the unit tensor, $\chi^{\text {iono }}$ and $\chi^{\text {torus }}$ are the ratios of the ions and neutral particle masses $\left(m_{i} / m_{n}\right)$ in the ionosphere and in the Io torus, respectively (we assume $\chi^{\text {iono }}=1$ and $\left.\chi^{\text {torus }}=1\right)$ and $U$ is the total energy $\left(U=\frac{\rho v^{2}}{2}+\frac{p}{\gamma-1}+\frac{B^{2}}{2 \mu_{0}}\right)$. Note that the mass of the ions does not explicitly appear in equations (4) to (7). An explicit assumption about the ion mass is only needed when number densities instead of mass densities and temperatures instead of internal energies are being displayed, which is not the case in this paper. The adiabatic index $\gamma$ is $5 / 3$. The velocity of the fluid is $\mathbf{v}=\frac{m_{e} \mathbf{v}_{\mathbf{e}}+m_{i} \mathbf{v}_{\mathbf{i}}}{m_{e}+m_{i}}$ In our model, the velocities of the neutral gas in the ionosphere ( $\left.\mathbf{v}_{\mathbf{n}}^{\text {iono }}\right)$ and in the Io torus ( $\left.\mathbf{v}_{\mathbf{n}}^{\text {torus }}\right)$ are prescribed: The neutral particles of the ionosphere are assumed to rigidly corotate with the planet, which means we neglect the slippage of the neutral atmosphere used, for example, by Huang and Hill [1989]; while the neutral particles of the Io torus are assumed to rotate at the local Keplerian velocity. As one can see in equation (5), momentum transfer is established between the atmosphere and the magnetosphere as a result of the collisions between the neutral particles and the ions $\left(\rho v_{\text {in }}\left(\mathbf{v}_{\mathbf{n}}^{\text {iono }}-\mathbf{v}\right)\right)$. The collision frequency of the ions with the neutral particles used in our model is spatially dependent. It is maximum at the inner boundary (at Jupiter), decreases with radial distance, and is zero outside of the ionospheric region. Equation (5) also shows how the momentum of the neutral gas is transferred to the plasma when the neutral particles are ionized in the Io torus $\left(\kappa \mathbf{v}_{\mathbf{n}}^{\text {torus }}\right)$. This term can, in principle, increase or decrease the bulk flow velocity of the plasma depending on the relative velocity of the neutral gas with respect to the plasma. In the Io torus, it should decelerate the plasma, because the velocity of the neutral particles (the Keplerian velocity) is lower than the velocity of the plasma (close to rigid corotation).

\subsection{Model Assumptions and Simplifications}

[20] Estimating the values of each term in the momentum equation in the ionosphere (see Table A3 in Appendix A), it can be shown that two terms, $\mathbf{j} \times \mathbf{B}$ and $\rho v_{i n}\left(\mathbf{v}-\mathbf{v}_{\mathbf{n}}\right)$, are orders of magnitude more important than the other terms. The following force balance is thus a good approximation in the ionosphere:

$$
\mathbf{j} \times \mathbf{B}=\rho v_{\text {in }}\left(\mathbf{v}-\mathbf{v}_{\mathbf{n}}\right) \text {. }
$$

This ionospheric force balance is also assumed in the analytic models of Hill [1979, 2001], Cowley and Bunce 
[2001], Cowley et al. [2002, 2003], and Nichols and Cowley [2003]. It shows how the ion-neutral collisions and the magnetic Lorentz forces are approximately balanced in the ionosphere.

[21] The $\nabla \times\left(-\frac{\mathbf{j} \times \mathbf{B}}{n e}\right)$ and $\frac{\mathbf{B}}{\mu_{0}} \cdot \nabla \times\left(-\frac{\mathbf{j} \times \mathbf{B}}{n e}\right)$ terms in equations (6) and (7), respectively, which are referred as Hall terms in these equations, play an important role in the ionosphere, i.e., where the magnetic field and the current are large. Making use of approximation (8), the Hall term will be approximated by $\nabla \times\left(-\frac{m_{i} v_{i n}\left(\mathbf{v}-\mathbf{v}_{\mathbf{n}}\right)}{e}\right)$ and $\frac{\mathbf{B}}{\mu_{0}} \cdot \nabla \times\left(-\frac{m_{i} v_{i n}\left(\mathbf{v}-\mathbf{v}_{\mathbf{n}}\right)}{e}\right)$ in the induction and energy equations of our model, respectively. This assumption should allow us to simulate the effects of the currents in the ionosphere without having to solve the Hall MHD equations. It should be noted that by using this approximation, we neglect the Hall term outside of the ionosphere (since $v_{i n}$ is zero there) and that our model is thus not a Hall-MHD model.

[22] Equations (4) to (7) are solved by the MessagePassing Interface-Adaptive Mesh Refinement Versatile Advection Code (MPI-AMRVAC) [van der Holst and Keppens, 2007; Keppens et al., 2012], which is the adaptive mesh refinement version of the Versatile Advection Code (VAC) [Tóth, 1996]. The solver is based on a TotalVariation-Diminishing Lax-Friedrichs numerical scheme and is used in a finite volume setting. In addition, we use the magnetic field splitting method developed by Tanaka [1994]. The MPI-AMRVAC and VAC codes have already been successfully applied to various problems, e.g., solar wind and coronal mass ejections [Chané et al., 2008; van der Holst et al., 2007], extragalactic jets [Keppens et al., 2008; Meliani et al., 2008], or supernova remnants [Schure et al., 2009].

[23] We use two different nonequidistant spherical meshes: a mesh to test the model (mesh 1) and a refined mesh to perform one high resolution run (mesh 2). For mesh 1, the cells are the smallest at the inner boundary. For mesh 2, 3.5 times more cells are used, and the resolution is increased in the current sheet: The smallest cells are located in a domain where the radial distance is between $25 \mathrm{R}_{\mathrm{J}}$ and $76.5 \mathrm{R}_{\mathrm{J}}$ and the latitude between $-11^{\circ}$ and $11^{\circ}$. For both meshes, the domain extends between $4.5 \mathrm{R}_{\mathrm{J}}$ and $189 \mathrm{R}_{\mathrm{J}}$, and the cells have a size of $0.5 \mathrm{R}_{\mathrm{J}}$ at the inner boundary.

[24] The inner boundary of our current numerical model is located at $4.5 \mathrm{R}_{\mathrm{J}}$, instead of $1 \mathrm{R}_{\mathrm{J}}$, because in timeaccurate MHD computations based on explicit time-stepping schemes, the time step is limited by the Courant-FriedrichsLewy condition. Regions with a very high magnetic field and low plasma density (i.e., a very high Alfvén speed) slow down the computations significantly. If we moved the inner boundary closer to Jupiter, then the runs would thus become unfeasibly slow. At the inner boundary, which is below the ionosphere, we set the radial velocity and the radial current density to zero. The radial current density is set to zero by simply fixing the azimuthal and the latitudinal component of the magnetic field to the Jovian dipole field. Since the radial velocity is set to zero, no plasma can flow through the boundary. As a result, the mass loading in the inner magnetosphere is entirely controlled by the source term used for the Io torus and not by the boundary conditions. Fixing the radial current density to zero at the boundary is very important since the current systems should be closed in our numerical domain (in our ionospheric region, located above the inner boundary) and should not continue artificially through the surface of the planet. Boundary conditions where the radial current is not set to zero might lead to nonphysical results due to uncontrolled $\mathbf{j} \times \mathbf{B}$ forces, e.g., supercorotation in the magnetosphere.

[25] At the outer boundary, open boundary conditions are applied at the nightside, while on the dayside, the solar wind is prescribed as follows:

$$
\begin{array}{ll}
\rho=1 \mathrm{amu} \mathrm{cm}{ }^{-3}, & v_{x}=-300 \mathrm{~km} / \mathrm{s}, \\
T=5000 \mathrm{~K}, & B_{z}=0.78 \mathrm{nT},
\end{array}
$$

where $z$ is the direction of the rotation axis of Jupiter (pointing north) and $x$ is directed to the Sun. These parameters describe a solar wind with an antiparallel interplanetary magnetic field (IMF) and a strong (but realistic) dynamic pressure (our dynamic pressure is similar to the average value for disturbed solar wind conditions computed by Joy et al. [2002]).

[26] Because the centrifugal force at the inner boundary $\left(4.5 \mathrm{R}_{\mathrm{J}}\right)$ is larger than gravity, we need to adjust the gravity in order to have an ionosphere bound to the planet. To do so, we use an artificially enhanced $G M_{J}$ which is 55 times stronger than the normal value. As a result, the centrifugal and gravitational forces are in equilibrium at 8.5 $\mathrm{R}_{\mathrm{J}}$. This enhancement only has a minor effect on the magnetosphere since gravity decreases rapidly with radial distance and plays an important role only close to the planet. We adjust the ion-neutral collision frequency in the ionosphere to mimic its key physical properties, e.g., its Pedersen and Hall conductances. Our ionospheric region as an extension of $4 \mathrm{R}_{\mathrm{J}}$ : the collision frequency is maximal at $4.5 \mathrm{R}_{\mathrm{J}}$ (at $R_{0}$, where $v_{i n}=v_{i n 0}$ ) and decreases linearly (due to numerical reasons) up to $8.5 \mathrm{R}_{\mathrm{J}}$ (at $R_{\mathrm{iono}}^{\max }$, where $\left.v_{i n}=0\right)$; beyond this point, we are outside the ionospheric region, and the collision frequency is set to zero. The Pedersen ionospheric conductivity is given by the following expression [Pedersen, 1927]:

$$
\sigma=\frac{e n}{B} \frac{\omega_{i} v_{i n}}{\omega_{i}^{2}+v_{i n}^{2}}
$$

where $\omega_{i}$ is the ion gyrofrequency. Integrating $\sigma$ along the field lines gives the ionospheric conductance. This expression shows how the ionospheric conductance can be changed by modifying the ion-neutral collision frequency, which is a free parameter in the model. As a further simplification, we currently neglect the $10^{\circ}$ tilt of the dipole axis relative to the spin axis.

[27] In our model, we intend to get a clear separation between the ionospheric region and the Io torus, which with our current mesh cannot be obtained for a torus located at $5.9 \mathrm{R}_{\mathrm{J}}$. Therefore, we locate the Io torus at $10 \mathrm{R}_{\mathrm{J}}$. Since the position of our torus is radially inside of the position of the corotation breakdown, this does not strongly affect the results as the transport in the $r<10 \mathrm{R}_{\mathrm{J}}$ region contributes much less to the field aligned magnetosphere-ionosphere coupling system than for $r>10 \mathrm{R}_{\mathrm{J}}$.

[28] The extra plasma generated by Io is introduced in the equations by means of a spatially dependent mass density loading rate, $\kappa$, which is strong in the center of the Io torus, 
CHANÉ ET AL.: JUPITER'S MAGNETOSPHERE

Table 1. Position of the Bow Shock, Magnetopause, and Corotation Breakdown for the Five Runs ${ }^{\mathrm{a}}$

\begin{tabular}{lcccccc}
\hline Run & Mass Loading & Max. Coll. Freq. & Conductance & Bow Shock & Magnetopause & Corot. Breakdown \\
\hline A & $15 \mathrm{t} / \mathrm{s}$ & $1.025 \mathrm{~s}^{-1}$ & $0.135 \mathrm{~S}$ & $77 \mathrm{R}_{\mathrm{J}}$ & $62 \mathrm{R}_{\mathrm{J}}$ & $20.6 \mathrm{R}_{\mathrm{J}}$ \\
B & $3 \mathrm{t} / \mathrm{s}$ & $1.025 \mathrm{~s}^{-1}$ & $0.056 \mathrm{~S}$ & $64 \mathrm{R}_{\mathrm{J}}$ & $50 \mathrm{R}_{\mathrm{J}}$ & $21.5 \mathrm{R}_{\mathrm{J}}$ \\
C & $1 \mathrm{t} / \mathrm{s}$ & $1.025 \mathrm{~s}^{-1}$ & $0.068 \mathrm{~S}$ & $61 \mathrm{R}_{\mathrm{J}}$ & $47 \mathrm{R}_{\mathrm{J}}$ & $25.4 \mathrm{R}_{\mathrm{J}}$ \\
D & $1 \mathrm{t} / \mathrm{s}$ & $0.5125 \mathrm{~s}^{-1}$ & $0.035 \mathrm{~S}$ & $60 \mathrm{R}_{\mathrm{J}}$ & $45 \mathrm{R}_{\mathrm{J}}$ & $20.9 \mathrm{R}_{\mathrm{J}}$ \\
E & $1 \mathrm{t} / \mathrm{s}$ & $0.205 \mathrm{~s}^{-1}$ & $0.1 \mathrm{~S}$ & $73 \mathrm{R}_{\mathrm{J}}$ & $69 \mathrm{R}_{\mathrm{J}}$ & $30.1 \mathrm{R}_{\mathrm{J}}$ \\
\hline
\end{tabular}

${ }^{\text {a }}$ The values of the Io torus mass loading rate and of the maximal ionospheric ion-neutral collision frequency $\left(v_{i n 0}\right)$ used, as well as the resulting ionospheric conductance (for one hemisphere) are also given. The solar wind dynamic pressure in the model runs is $0.15 \mathrm{nPa}$, and the magnetic field is purely northward, with $B_{z}=0.78 \mathrm{nT}$. Max. Coll. Freq., maximum collision frequency; Corot., corotation.

decreases smoothly with distance from the center, and is zero outside of the torus:

$$
\begin{cases}\kappa=\kappa_{0} \frac{1-\cos \left(\pi \frac{h-d}{h}\right)}{2} & \text { if } d<1 R_{\mathrm{J}} \\ \kappa=0 & \text { otherwise, }\end{cases}
$$

where $d$ is the distance from the center of the torus, and $h$ is half the width of the torus; $h$ is fixed to $1 \mathrm{R}_{\mathrm{J}}$, and $\kappa_{0}$ is a fixed value at the center of the torus.

[29] The characteristics of our model can be summarized as follows: a global one-fluid MHD model of the interactions between the solar wind and Jupiter's magnetosphere with an extended ionosphere (between $4.5 \mathrm{R}_{\mathrm{J}}$ and $8.5 \mathrm{R}_{\mathrm{J}}$ ) where ion-neutral collisions take place, with an Io torus (located at $10 \mathrm{R}_{\mathrm{J}}$ instead of $5.9 \mathrm{R}_{\mathrm{J}}$ ) where mass loading occurs, with an enhanced gravity to obtain an ionosphere bound to the planet, and with no dipole tilt included. The location of the inner boundary, the size of our ionospheric region, and the position of our Io torus are shown in Figure 1 where they are drawn to scale. In this model, the two key characteristics of the Jovian magnetosphere, namely the ionospheric conductance and the Io torus mass loading, are controlled.

\section{Numerical Model: Setup and Results}

[30] In this section, we present results of five different numerical model runs. We first explain the numerical model setup and how the five runs differ (mesh, mass loading, and ionospheric conductance). We then show the general configuration of the magnetosphere in our model runs: magnetic field, density profile, velocity field, and current systems, and compare them with theoretical expectations and in situ measurements. To test the accuracy of our ionospheremagnetosphere coupling, we then compare the azimuthal velocity profiles in the five model runs with results from analytical models. Finally, we compare the obtained auroral pattern with observations.

\subsection{Numerical Model: Setup}

[31] Four runs (A, B, C, and D) are performed on mesh 1 and are used to test our magnetosphere-ionosphere coupling model by performing runs with different Io torus mass loading rates and different ionospheric conductances. Run $\mathrm{E}$ is performed on a better mesh (mesh 2), and the results obtained with this mesh will be studied in more details. The influence of the Io torus mass loading rate on our model is studied with runs $\mathrm{A}, \mathrm{B}$, and $\mathrm{C}$, where the total mass loading at Io is set to 15,3 , and $1 \mathrm{t} / \mathrm{s}$, respectively, while the maximal ionospheric ion-neutral collision frequency is $v_{i n 0}=1.025 \mathrm{~s}^{-1}$. The amount of mass loading in the Io torus is subject to some uncertainties. Several authors have derived this rate and found different values: see review papers from Thomas et al. [2004] and Bagenal and Delamere [2011]. For example, Bagenal and Delamere [2011] derived values between 260 and $1400 \mathrm{~kg} / \mathrm{s}$ for the plasma production rate. Therefore, we generate runs which reflect the various estimates that occur in the literature. We use a mass loading rate of $3 \mathrm{t} / \mathrm{s}$ in run $\mathrm{B}$ and $1 \mathrm{t} / \mathrm{s}$ in runs $\mathrm{C}, \mathrm{D}$, and $\mathrm{E}$. In run $\mathrm{A}$, a rate of $15 \mathrm{t} / \mathrm{s}$ is used. The later value is unrealistically high and was chosen to test our magnetosphere-ionosphere coupling for very high mass loading rates.

[32] The influence of the ionospheric conductance on our model will be studied by comparing runs $\mathrm{C}, \mathrm{D}$, and $\mathrm{E}$, where the total mass loading in the Io torus is fixed to $1 \mathrm{t} / \mathrm{s}$ and where the maximal collision frequency $\left(v_{i n 0}\right)$ is set to $1.025 \mathrm{~s}^{-1}$ in run $\mathrm{C}$, to $0.5125 \mathrm{~s}^{-1}$ in run $\mathrm{D}$, and to $0.205 \mathrm{~s}^{-1}$ in run $\mathrm{E}$. As a result, while the ionospheric conductance is $0.068 \mathrm{~S}$ in run $\mathrm{C}$, it is only $0.035 \mathrm{~S}$ in run $\mathrm{D}$. The ionospheric conductance is higher in run $\mathrm{E}(0.1 \mathrm{~S})$ even though the collision frequency is lower: This is because the density in the ionospheric region is higher in run $\mathrm{E}$, due to the lower numerical diffusion (run $\mathrm{E}$ is performed on a better mesh). Even though the ionospheric conductance of Jupiter is not strongly constrained by models and observations, these values are low compared to values of Strobel and Atreya [1983], who estimated that the ionospheric conductance should be between $0.2 \mathrm{~S}$ and $10 \mathrm{~S}$. In addition, Jupiter's ionospheric conductance displays spatial variabilities and should be higher in the auroral regions due to electron precipitation [see for instance Nichols and Cowley, 2004; Tao et al., 2010]; the enhancement of the conductivity caused by electron precipitation is not included in our model. At Jupiter, the linear approximation to the Knight currentvoltage relation cannot be used, and field-aligned potentials should be treated self-consistently [Ray et al., 2009, 2010, 2012]. We neglect this effect in our model and use $\frac{j_{\|}}{B}$ to visualize the aurorae. It should be mentioned that, in our computations, the mass loading rate used in the Io torus influences indirectly the Jovian ionospheric conductance (as one can see in Table 1). This is because the plasma density in the ionosphere increases slightly when the Io torus mass loading rate is high (due to numerical diffusion), which thus tends to increase the ionospheric conductivity (equation (9)).

\subsection{Computation Results of Numerical Model}

[33] Figure 2 shows the density and the magnetic field in the noon-midnight meridian for run $\mathrm{E}$, after it reached a quasi steady state. All the results presented in this paper were obtained when such a quasi steady state was reached. It 


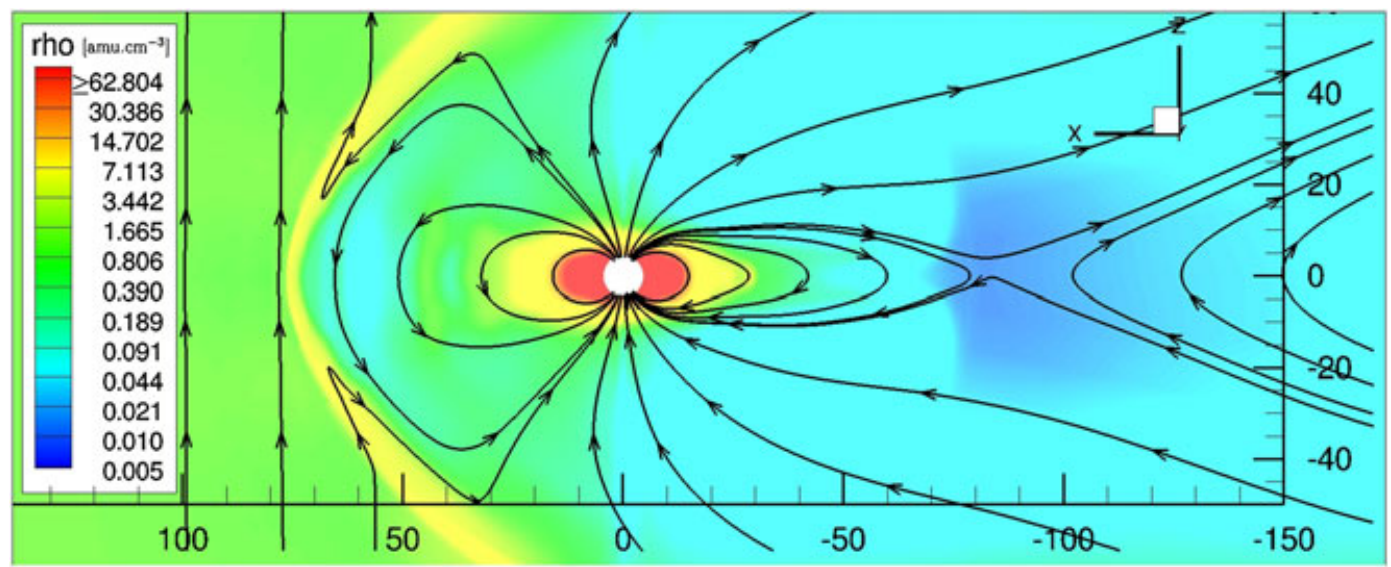

Figure 2. Density contours in the noon-midnight meridian (side view) for run E. The magnetic field lines are shown in black. The solar wind comes from the left.

means that changes are still observed, but the global configuration is not changing anymore on a longer timescale. Blobs of high density, for instance, tend to be ejected episodically in the magnetotail. Long periods of time are needed to reach such quasi steady state configurations: It took, for example, more than 10 rotation periods for run $\mathrm{A}$.

[34] One can see in Figure 2 that the IMF, which comes from the right, is antiparallel to the Jovian magnetic field. This results in an open magnetosphere with field lines connecting the Jovian ionosphere to the IMF above the poles. For runs with a parallel IMF (not shown here), we obtain a closed magnetosphere. In this figure, we see how the interaction between the solar wind and Jupiter's magnetosphere produces elongated magnetic field lines in the tail and compressed field lines on the dayside, with a magnetopause located at $69 \mathrm{R}_{\mathrm{J}}$ and a bow shock at $73 \mathrm{R}_{\mathrm{J}}$. Based on in situ measurements, Joy et al. [2002] showed that the range of subsolar magnetopause positions is between $50 R_{J}$ and $100 R_{J}$, while the range of subsolar bow shock positions is between $55 R_{J}$ and $125 R_{J}$. To be precise, they found a bimodal probability distribution for the magnetopause location and for the bow shock location (even though a single distribution function description cannot be ruled out for the later). The most probable standoff distances are $63 \mathrm{R}_{\mathrm{J}}\left(\sigma=4 \mathrm{R}_{\mathrm{J}}\right)$ and $92 \mathrm{R}_{\mathrm{J}}\left(\sigma=6 \mathrm{R}_{\mathrm{J}}\right)$ for the magnetopause; and $73 \mathrm{R}_{\mathrm{J}}\left(\sigma=10 \mathrm{R}_{\mathrm{J}}\right)$ and $108 \mathrm{R}_{\mathrm{J}}\left(\sigma=10 \mathrm{R}_{\mathrm{J}}\right)$ for the bow shock. Since we use a dense solar wind and an antiparallel IMF, our magnetopause and bow shock are closer to Jupiter than the average values. It can be seen in Figure 2 that the equatorial confinement of the plasma is characterized by a scale height which is approximately three times larger than what is usually observed in the magnetosphere. This is due the resolution. With a finer mesh, we would obtain a better equatorial confinement. The reason is that a finite numerical resolution generates numerical resistivity and numerical viscosity. The associated dissipative effects convert electromagnetic field energy and kinetic energy into heat, and the resultant heating rate significantly enhances the internal energy of the magnetosphere. This numerical effect resembles to some extent the real physical heating of the magnetosphere. Based on the observed temperature/energy density profiles in Jupiter's magnetosphere [e.g., Frank et al., 2002; Bagenal and Delamere, 2011], such a heating processes is necessary and could, for example, be caused by dissipation of the small-scale turbulent magnetic fluctuations in Jupiter's magnetosphere [Saur, 2004]. We do not additionally include such a heating term in our energy equation due to the numerical dissipation already present in our model.

[35] The results presented in Figure 2 for run E are globally similar to the results obtained for runs A, B, C, and D, even though some differences exist. For instance, the position of the bow shock and of the magnetopause which are summarized in Table 1 or the current sheet which is less visible in runs A, B, C, and D due to the use of a coarser mesh. One can see that, the higher the mass loading (or the higher the ionospheric conductance), the farther out are the magnetopause and the bow shock. For strong mass loading rates, more plasma is present in the magnetosphere, and the centrifugal force and plasma pressure opposing the solar wind ram pressure are stronger. Consequently, the bow shock and the magnetopause are located farther out. When the ionospheric conductance is low, the momentum transfer between the planet and the magnetosphere is low; the plasma velocity

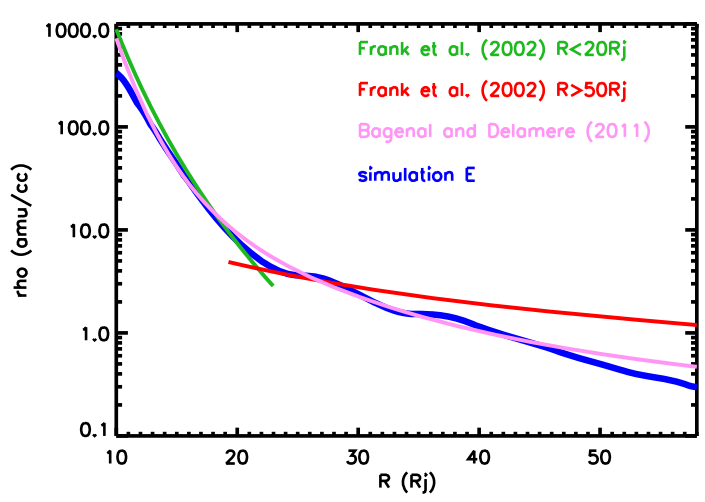

Figure 3. Density in the equatorial plane: Comparison between our results (shown in blue), the fits to observations by [Frank et al., 2002] (in green and red), and Bagenal and Delamere [2011] (in purple). Our results are shown for run E and are averaged over all local times. For the Frank et al. [2002] and Bagenal and Delamere [2011] models, we assumed a mass of $\sim 22$ amu for the ions. 


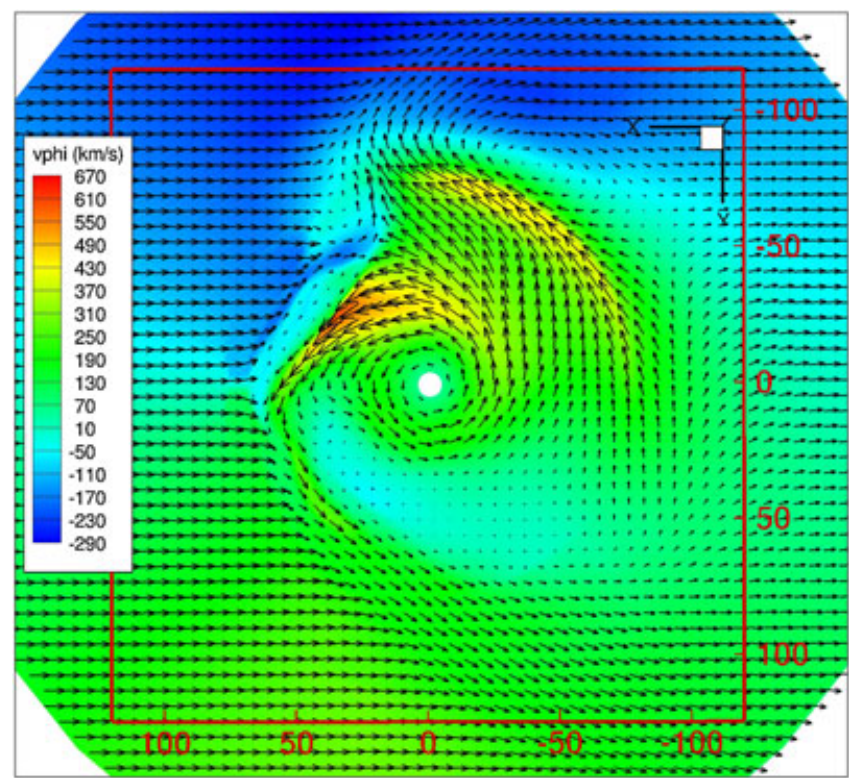

Figure 4. Velocity in the equatorial plane (top view) for run E. The black arrows display the velocity in this plane; the color contours the azimuthal velocity. The solar wind comes from the left.

and, consequently, the centrifugal force in the current sheet are then lower. The bow shock and the magnetopause are therefore located closer to Jupiter.

[36] Figure 3 compares the density we obtained with the model of Frank et al. [2002] and the model of Bagenal and Delamere [2011]. The density of Frank et al. [2002] has been obtained during the G8 orbit of Galileo, when the spacecraft was in the magnetotail. The Bagenal and Delamere [2011] density profile is based on Voyager 1 (1979) and Galileo (1996-2003) data. It can be seen in this figure that the density profile obtained with run $\mathrm{E}$ is realistic.

[37] Figure 4 displays the velocity in the equatorial plane for run E. It shows how the solar wind plasma is deviated around the magnetopause and how the magnetospheric plasma rotates around the planet. In this figure, one can see the trajectory of the Iogenic plasma; and how, after numerous rotations around the planet, it is ejected in the tail. The radial velocity is not always and everywhere positive in the magnetosphere. When a quasi steady state is reached, some regions of the magnetosphere (sometimes large regions) episodically display negative radial velocities. While moderate negative radial velocities (between 0 and $-100 \mathrm{~km} / \mathrm{s}$ ) occasionally occur at all local times, strong inward flows $(-100 \mathrm{~km} / \mathrm{s}$ or stronger) occur mostly in two specific regions: (1) around 9:00 LT where the rotating plasma encounters the magnetopause (usually $-150 \mathrm{~km} / \mathrm{s}<v_{r}<-100 \mathrm{~km} / \mathrm{s}$, but $v_{r}$ can be as low as $300 \mathrm{~km} / \mathrm{s}$ ), and (2) in the magnetotail (where $v_{r}$ can reach $-480 \mathrm{~km} / \mathrm{s}$, but usually lies between $-100 \mathrm{~km} / \mathrm{s}$ and $-200 \mathrm{~km} / \mathrm{s}$ ). These strong inward flows in the tail last for approximately $12 \mathrm{~h}$ and can affect very large regions: at their largest, spreading between 40 and $80 \mathrm{R}_{\mathrm{J}}$, and between 21:00 LT and 4:00 LT. Even though these events do not display a specific periodicity, there is usually approximately $30 \mathrm{~h}$ between two events. During these periods of strong inward flows, the closed magnetic field line region in the tail usually shrinks. These events are sometimes, but not always, related to ejection of large plasmoids in the tail. The large plasmoids are usually located in the postmidnight sector and can be very large (the largest extends between 23:00 LT and 7:00 LT).

[38] One can see in Figure 4 that the azimuthal velocity is higher on the dawnside than on the duskside. This is in agreement with in situ measurements [see, for instance, Woch et al., 2004, Figure 6]. In addition, the current sheet is also thicker on the duskside than on the dawnside (not shown in the figure), which is also in agreement with measurements [e.g., Khurana et al., 2004, Figure 24.20].

[39] Figure 5 shows the radial velocity in the equatorial plane as a function of radial distance; it compares the results of run $\mathrm{E}$ with the profiles derived by Bagenal and Delamere [2011], for mass loading rates of 0.26 and $1.4 \mathrm{t} / \mathrm{s}$. The radial velocity in run $\mathrm{E}$ (mass loading rate of $1 \mathrm{t} / \mathrm{s}$ ) lays between these two curves almost everywhere and is realistic. For this run, the transport time between $10 \mathrm{R}_{\mathrm{J}}$ (where the mass loading occurs in the computation) and $40 \mathrm{R}_{\mathrm{J}}$ is $41 \mathrm{~h} 21 \mathrm{~min}$. The plasma remains $30 \mathrm{~h} 53 \mathrm{~min}$ between 10 and $20 \mathrm{R}_{\mathrm{J}}, 6 \mathrm{~h}$ $56 \mathrm{~min}$ between 20 and $30 \mathrm{R}_{\mathrm{J}}$, and $3 \mathrm{~h} 32 \mathrm{~min}$ between 30 and $40 \mathrm{R}_{\mathrm{J}}$. At $10 \mathrm{R}_{\mathrm{J}}$, the radial velocity is 50 times lower than the azimuthal velocity, 12 times lower at $20 \mathrm{R}_{\mathrm{J}}$, and 5 times lower at $30 \mathrm{R}_{\mathrm{J}}$. The azimuthal and radial velocity becomes comparable at a radial distance of $42 \mathrm{R}_{\mathrm{J}}$. The mesh we use is too coarse to properly resolve the small scales associated with the Io torus. This explains why, at the position where the mass loading occurs $\left(10 \mathrm{R}_{\mathrm{J}}\right)$, our density is too low, and our radial velocity too high.

[40] The corotation enforcing current system can clearly be seen in our model (Figure 6). One can see in this figure how the radial currents in the equatorial plane are generated at the position of the corotation breakdown and beyond. The field-aligned currents that connect the corotation breakdown to the main oval are also clearly visible. The presence of this current system in the computational results is essential to study correctly the main auroral oval.

\subsection{Location of the Corotation Breakdown}

[41] Since the azimuthal velocity profile, and in particular the location of the corotation breakdown, is of tremendous

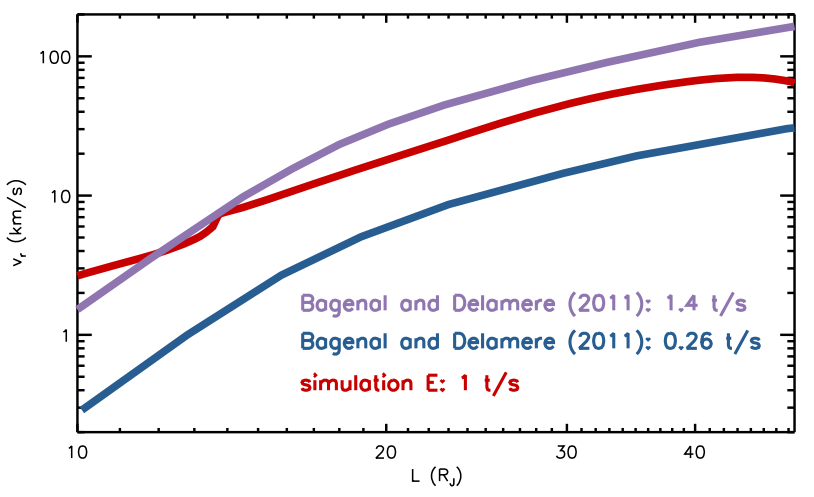

Figure 5. Radial velocity in the equatorial plane: Comparison between our results (shown in red) and profiles derived by Bagenal and Delamere [2011] for mass loading rates of $1.4 \mathrm{t} / \mathrm{s}$ (in purple) and $0.26 \mathrm{t} / \mathrm{s}$ (in blue). Our results are shown for run $\mathrm{E}$ and are averaged over all local times. 


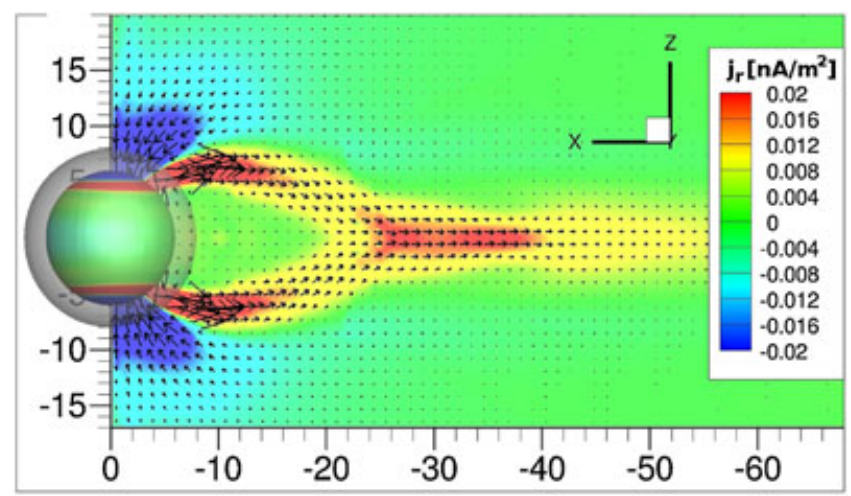

Figure 6. Corotation enforcing current system on the nightside of Jupiter in the noon-midnight meridian (side view) for run $E$. The radial current is shown with color contours, and the current in the same plane (i.e., $j_{\phi}$ is not shown) is represented by the black arrows. In addition, the radial current is also displayed in the ionospherical region, on a sphere at $6 R_{J}$, and a transparent sphere at $8 R_{J}$ shows the extend of the ionospherical region. Note that the corotation breakdown (as defined by Hill [1979], 75\% of rigid corotation) in this plane occurs at $-32 \mathrm{R}_{\mathrm{J}}$ but that the plasma starts to subcorotate at approximately $-22 \mathrm{R}_{\mathrm{J}}$.

importance for Jupiter's magnetosphere and for the main auroral oval, we examine how the azimuthal velocity evolves as a function of radial distance. We compare the azimuthal velocity profiles of the five runs with the model of Hill [1979, 2001] and Saur et al. [2004a]. Even though the model of Saur et al. [2004a] was developed for Saturn, it includes both mass loading and radial transport and can be readily applied to Jupiter. It thus allows more detailed comparisons with our results where these two phenomena are also included. Hill [1979, 2001] only includes the mass transport but not the mass loading process in this model.

[42] With this model, an analytic solution can be found, which gives the azimuthal velocity profiles as a function of radial distance for any given mass loading and ionospheric conductance. Solving equation (6) from Saur et al. [2004a], we compare the results with our numerical model in Figure 7. Our model output are in good agreement with the predicted results. This is specially true close to Jupiter (up to $\sim 20 \mathrm{R}_{\mathrm{J}}$ ), where the magnetic field is strongly dipolar. Farther out, when the magnetic field cannot be described by a dipole field anymore, a comparison with these analytical models is less pertinent, because these theoretical models assume a dipole field. The local decrease of the plasma velocity in the Io torus due to ionization of "slow" neutral particles is also visible in our results, even though this effect seems to be less prominent than in the model of Saur et al. 2004a]. This small discrepancy between the analytical models and the computations may be caused by numerical viscosity due to the relatively small spatial scale of the Io torus. This effect may prevent a more pronounced corotation lag in the torus to occur. The corotational lags in runs A, B, C, D, and $\mathrm{E}$ are $4.1 \%, 2.2 \%, 0.5 \%, 1.2 \%$, and $0.6 \%$, respectively.

[43] The azimuthal velocity profiles for the five runs are also shown in Figure 8, where they are compared with in situ measurements from Voyager I. One can see that our results are in good agreement with the measurements. In our computations, as expected, the higher the mass loading (or the lower the ionospheric conductance), the lower the azimuthal velocity. The location of the corotation breakdown is then closer to the planet (see Table 1 for the exact location of the corotation breakdown. Note that we consider, similar to Hill [1979], that the corotation breaks down when the azimuthal plasma velocity decreases down to $75 \%$ of rigid corotation.).

[44] These results show that our ionosphere model (with ion-neutral collisions in an extended area) generates realistic azimuthal velocity profiles. We find that our oversized ionosphere still produces reasonable velocity profiles, as long as we have a correct height-integrated Pedersen conductivity. This is, to our knowledge, the first time that azimuthal velocity profiles from global MHD numerical models are directly compared with theory and observations. In a similar way, the unrealistic position of the Io torus should not be an issue as long as the total mass loading is chosen correctly, and the torus is situated inside the location of the corotation breakdown. Even though our model is not realistic close
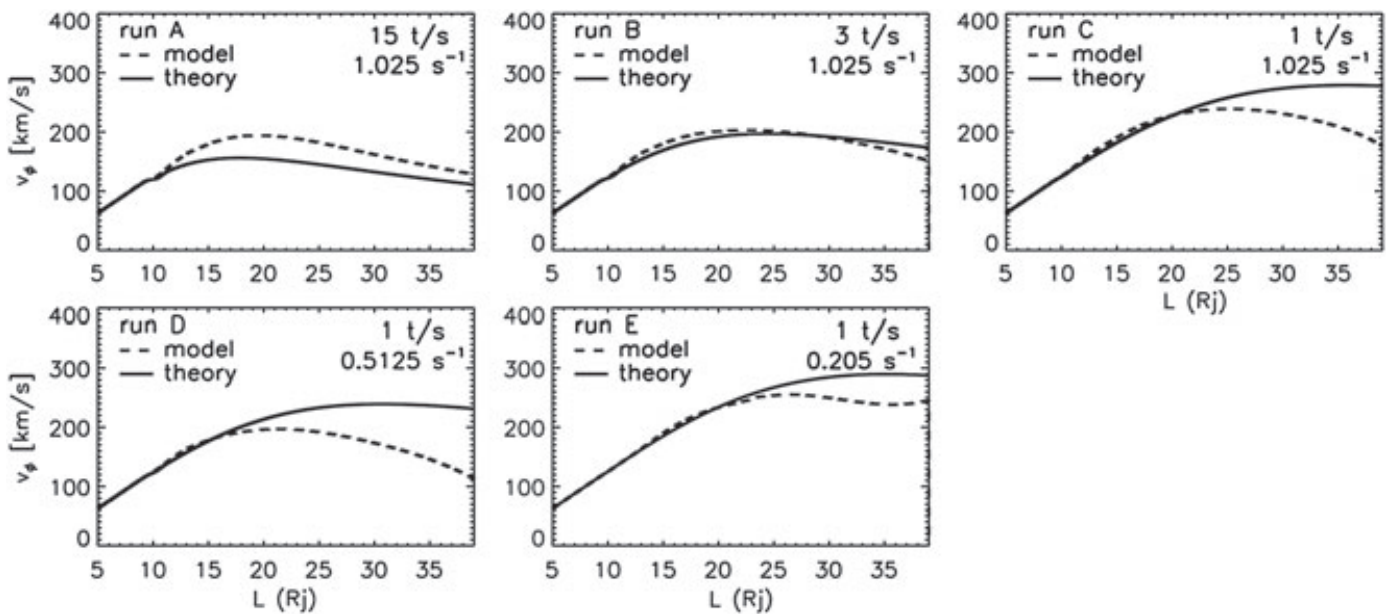

Figure 7. Comparison between the analytic solutions (solid lines) and the computations (dashed lines). Shown is the azimuthal velocity in the equatorial plane as a function of radial distance for all runs. The values were averaged over a rotation period and over all local times. 


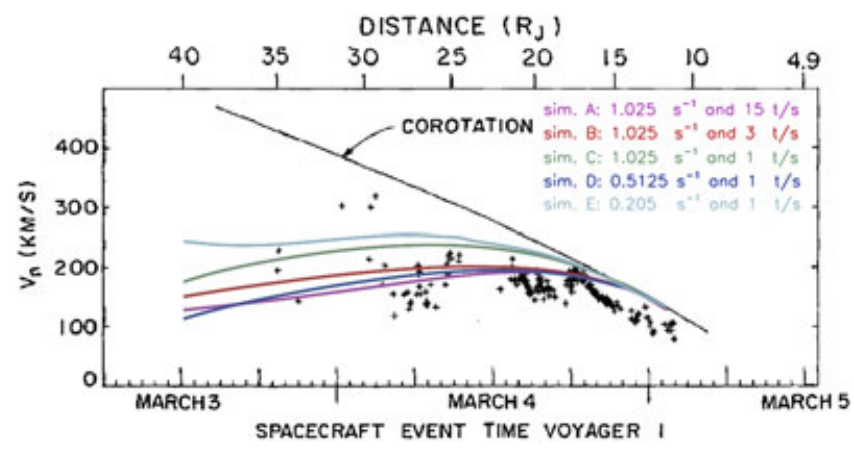

Figure 8. Azimuthal velocity as a function of radial distance: Comparison between Voyager I measurements (black plus signs) and computational results (color lines). The model run output values are overplotted on top of Figure 6.24 from Khurana et al. [2004] which was adapted from $\mathrm{McNutt}$ et al. [1981]. The model values are shown for different Io torus mass loading rates and ionospheric ionneutral collision frequencies. The black line represents rigid corotation. For the computations, the values given are for the equatorial plane and were averaged over a rotation period and over all local times.

to Jupiter, it is able to reproduce correctly the momentum transfer from the planet to the collisionless plasma of the current sheet caused by the ionosphere-magnetosphere coupling, as well as the deceleration of the plasma due to the outward transport of the plasma produced in the Io torus. This is a prerequisite to study the Jovian magnetosphere and the aurorae using global 3-D MHD models.

[45] Since, according to the theory, the location of the corotation breakdown is strongly related with the current system responsible for the aurorae, it is interesting to study the auroral structures in our model. This will be done in the next section.

\subsection{Investigation of the MI Coupling Currents and Aurorae}

[46] In our computations, the total radial current flowing from the magnetosphere to the ionosphere is equal to the total radial current flowing from the ionosphere to the magnetosphere. This shows that no current is lost through the inner boundary. The total current flowing into and out of the ionosphere is $48.7 \mathrm{MA}$ for run E. This value is comparable with 60 MA derived in Khurana et al. [2004]. Our value can also be compared with the maximum azimuth-integrated total current derived by Nichols and Cowley [2003]: i.e., $12 \mathrm{MA}$ when they consider a dipole field model and $73 \mathrm{MA}$ for the current sheet magnetic field model.

[47] In the acceleration region above Jupiter's ionosphere, electrons are accelerated to high velocities. When these electrons precipitate in the ionosphere, aurorae are generated. The larger the parallel currents density, the more electrons have to be accelerated once a certain threshold current density is reached [see Knight, 1973]. Consequently, regions of high upward parallel currents in the ionosphere are associated with the aurorae. Since $\frac{j_{\|}}{B}$ is constant along a field line, it is often used as proxy for the aurorae. The magnitude of $\frac{j_{\|}}{B}$ can be projected from our ionosphere down to $1 \mathrm{R}_{\mathrm{J}}$ by following dipole field lines. The results for the northern hemisphere are given for run $\mathrm{E}$ in Figure 9. In this figure, one can distinguish two areas of high parallel current density: (1) at the pole, the current is directed inward, and (2) around $15^{\circ}$ colatitude, the currents are directed outward, which corresponds to regions where electron aurorae are generated. The latter can be associated with the main auroral oval. Following the magnetic field lines from the position of the corotation breakdown (see values given in Table 1) to $1 \mathrm{R}_{\mathrm{J}}$, and assuming a dipole field below our inner boundary, one finds colatitudes of $15.1^{\circ}$, on average. Therefore, our main oval is, as expected, related to the position of the corotation breakdown. In addition, the magnitude of $\frac{j_{\|}}{B}$ and location of the main oval are in good agreement with the expectations (see, e.g., Clarke et al. [2004] where a value of $15^{\circ}$ is given for the colatitude of the main oval and a value of $10^{-4} \mathrm{~A} \mathrm{~m}^{-2} \mathrm{~T}^{-1}$ for $\left.\frac{j_{\|}}{B}\right]$.

[48] The values of $\frac{j \|}{B}$ can also be compared with the analytical model of Nichols and Cowley [2003]. For example, using their model, one finds values of $1.5 \times 10^{-4} \mathrm{~A} / \mathrm{m}^{2} / \mathrm{T}$ and $1.1 \times 10^{-5} \mathrm{~A} / \mathrm{m}^{2} / \mathrm{T}$ for the current sheet model and the dipole field model, respectively.

[49] The observations of Jupiter's far ultraviolet auroral emissions obtained with the Hubble Space Telescope [Radioti et al., 2008] suggest that a discontinuity in the main emission is located between 8:00 LT and 13:00 LT (the discontinuity was observed in 56 out of 64 images). It might seem surprising that the solar wind exerts an influence so deep inside the magnetosphere, but local time asymmetries can also be seen in the magnetic field of in situ measurements deep inside the magnetosphere [see, for instance, Khurana, 2001, Figures 5, 6, and 7]. The observations of Radioti et al. [2008] are supporting the in situ measurements of Khurana [2001]; who, using measurements of six different spacecraft, showed that the currents flowing in the equatorial plane should produce weaker aurorae at approximately the same position (see plate 6 in this article).

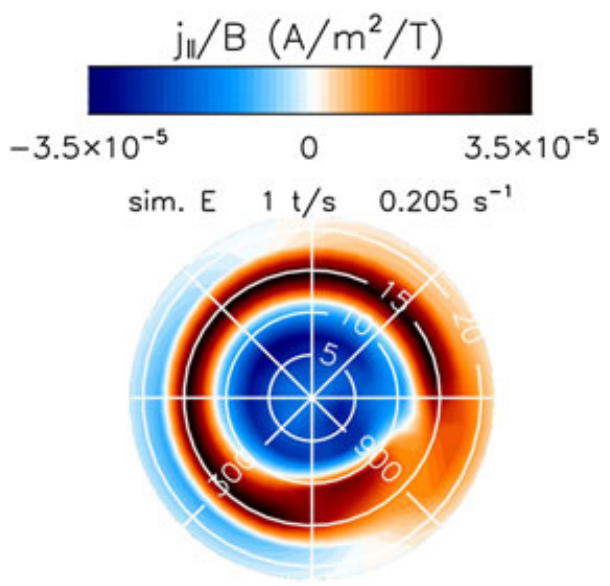

Figure 9. The color contours show $\frac{j_{\|}}{B}$ in the northern hemisphere at $1 \mathrm{R}_{\mathrm{J}}$ for run $\mathrm{E}$. The values of $\frac{j_{\|}}{B}$ were projected from our ionosphere to a sphere at $1 \mathrm{R}_{\mathrm{J}}$ by following the dipole magnetic field lines. The value of $\frac{j_{\|}}{B}$ was averaged over a rotation period. The colatitude and the local time are plotted in white on the figures; the dayside is located on the right. 


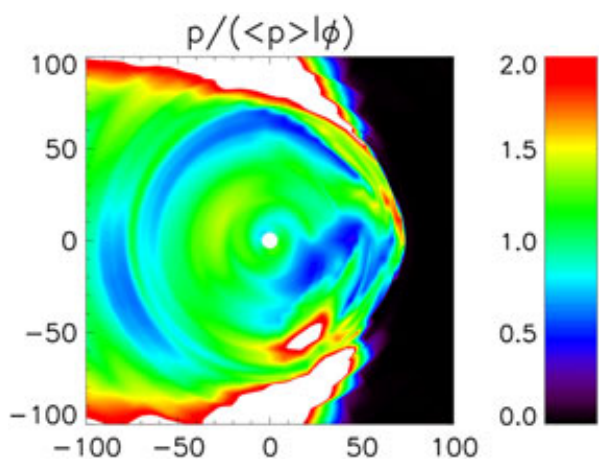

Figure 10. Pressure normalized with the pressure averaged for all local times in the equatorial plane (top view), values higher than two are shown in white. A large area with small values is visible on the pre-noon sector around $30 R_{J}$. These results are for run $\mathrm{E}$. The solar wind comes from the right.

[50] As can be seen in Figure 9, our model also produces a weaker main oval in the pre-noon sector. These weaker aurorae are associated with a weaker radial current in the equatorial plane at the position of the corotation breakdown in our computations. The radial current is weaker there, because the magnetic field lines are less bent in the azimuthal direction. This is due to a weaker decrease of the angular velocity with radial distance in the pre-noon sector (Figure 4). In our results, the angular velocity is higher in this region because the pressure gradient in the azimuthal direction is larger there (Figure 10). The pressure minimum displayed in Figure 10 is located at approximately $30 \mathrm{R}_{\mathrm{J}}$. Even though the pressure pattern displayed in Figure 10 tends to vary (e.g., ejection of plasmoids), the pressure minimum in the pre-noon sector is almost always present. This pressure minimum is mainly caused by the divergence/expansion of the flow $\nabla \cdot \mathbf{v}$ : When the plasma rotates around Jupiter, it is more confined on the dayside due to the presence of the magnetopause. When the plasma passes $\sim 18: 00 \mathrm{LT}$, it is not affected by the magnetopause, free to expend, and $\nabla \cdot \mathbf{v}$ is positive. Only once the plasma arrives around 9:00 LT, it is affected by the presence of the magnetopause, and $\nabla \cdot \mathbf{v}$ becomes negative. A positive $\nabla \cdot \mathbf{v}$ tends to adiabatically decrease the pressure. The pressure thus decreases when the plasma rotates around Jupiter on the nightside, reaches a minimum around 9:00 LT and then, due to the confinement caused by the magnetopause, increases again.

[51] In a nutshell, the thermal pressure is minimum around 9:00 LT due to the interaction between the rotating plasma and the magnetopause; as a result, the plasma is accelerated, and the azimuthal velocity is higher on the dawnside. This asymmetry implies that the magnetic field lines are less bent in the pre-noon sector, which prevents the formation of a high radial current in the equatorial plane, which causes the aurorae to be weaker there.

\section{Conclusions}

[52] In this article, we present a new 3-D global MHD model of the interaction between the solar wind and the Jovian magnetosphere. With this model, we numerically study the influence of the mass loading rates and of the ionospheric conductivities on Jupiter's magnetosphere. This model differs from the previous 3-D global MHD models by (1) including the Io torus mass loading inside the numerical domain, and (2) introducing an extended ionospherical region where collisions between the neutral gas and the plasma are explicitly taken into account. Consequently, our model allows us to control the solar wind conditions, the Io torus mass loading, and the ionospheric conductance.

[53] We demonstrate that, even though the model has disadvantages and includes simplifications pertaining to the region close to Jupiter (e.g., size of the ionospherical region, position of the Io torus, and gravity 55 times too high), it reproduces the expected theoretical azimuthal velocity profiles in Jupiter's magnetosphere for a large range of Io mass loading rate and ionospheric conductances. This was done by comparing our results with analytical models [Hill, 1979, 2001; Saur et al., 2004a]. The total current flowing in our computations between the ionosphere and magnetosphere as well as the local current densities agree well with measurements and analytical models. We use $\frac{j_{\|}}{B}$ as a proxy for the main oval emission and show that, in our computations, as expected, the corotation breakdown is associated with the main oval. The corotation enforcing current system, which is responsible for the main oval [Hill, 2001; Cowley and Bunce, 2001], is clearly visible in our model. The discontinuity in the main oval emission located on the pre-noon sector, which was observed for the first time by Radioti et al. [2008], is also present in our results. The results suggest that the corotation enforcing current system is weaker at this position because the azimuthal velocity does not decrease as steeply with radial distance, as it does at different locations. This is caused by a thermal pressure minimum located in the pre-noon sector in our model and produced by the position of the magnetopause and the rotation of the plasma around the planet. This hinders the expansion of the plasma around noon, but not for other local times, allowing the pressure to decrease while the plasma rotates around the planet until it reaches the pre-noon sector.

\section{Appendix A: Time Scales of Terms in MHD Equations}

[54] In the derivation of equations (4) to (7) from equations (1) to (3), several terms are neglected. We show here how the importance of each term is evaluated systematically by calculating its typical timescale; the terms displaying very long timescales are neglected. Since the extra terms added to the MHD equations (by introducing $v_{\text {in }}$ and $\left.\kappa\right)$ are only present in the ionosphere or in the Io torus, typical timescales are calculated in these two regions. To evaluate these timescales, we need typical values for all the variables present in our equations (e.g., a typical velocity in the ionosphere can be evaluated by taking the velocity associated with rigid corotation). Typical values for all the variables used in our equations as well as the explanations leading to their estimation are presented in Table A1. In Tables A2, A3, A4, and A5, we list all terms, which emerge in a rigorous derivation of the one-fluid MHD equations when source terms in Jupiter's ionosphere and the Io plasma torus are included. The complete one-fluid MHD equations leading to equations (4) to (7) (before simplifications) are 
Table A1. Typical Values for Each Variable

\begin{tabular}{|c|c|c|c|}
\hline & Ionosphere & Torus & $\begin{array}{l}\text { Explanations and } \\
\text { References }\end{array}$ \\
\hline$v(\mathrm{~m} / \mathrm{s})$ & 57,000 & 127,000 & $\begin{array}{l}\text { Rigid corotation at } \\
4.5 \mathrm{R}_{\mathrm{J}} \text { and } 10.0 \mathrm{R}_{\mathrm{J}}\end{array}$ \\
\hline$v_{n}(\mathrm{~m} / \mathrm{s})$ & 57,000 & 99,000 & $\begin{array}{l}\text { Rigid corotation and } \\
\text { Keplerian velocity }\end{array}$ \\
\hline$p_{i}(\mathrm{~Pa})$ & $2 \times 10^{-9}$ & $3.2 \times 10^{-8}$ & $\begin{array}{l}\text { Ionosphere: see }{ }^{\mathrm{a}} \text {; torus: } \\
\text { Kivelson et al. }[2004]\end{array}$ \\
\hline$p_{e}(\mathrm{~Pa})$ & $2 \times 10^{-9}$ & $2.4 \times 10^{-9}$ & $\begin{array}{l}\text { Ionosphere: see }{ }^{\text {a }} \text {; torus: } \\
\text { Kivelson et al. }[2004]\end{array}$ \\
\hline$T_{n}(\mathrm{~K})$ & 160 & 225 & $\begin{array}{l}\text { Yelle and Miller [2004]; } \\
\text { Ballester et al. [1994] }\end{array}$ \\
\hline$\nabla\left(\mathrm{m}^{-1}\right)$ & $1.4 \times 10^{-8}$ & $1.4 \times 10^{-8}$ & Typical lengths of $1 R_{J}$ \\
\hline$j\left(\mathrm{~A} / \mathrm{m}^{2}\right)$ & $10^{-9}$ & $1.1 \times 10^{-9}$ & Khurana et al. [2004], see \\
\hline$m_{i}(\mathrm{amu})$ & 1 & 22 & $\begin{array}{r}\text { Yelle and Miller [2004]; } \\
\text { Thomas et al. }[2004]\end{array}$ \\
\hline$m_{n}(\mathrm{amu})$ & 2 & 22 & $\begin{array}{l}\text { Yelle and Miller [2004]; } \\
\text { Thomas et al. [2004] }\end{array}$ \\
\hline$B(\mathrm{~T})$ & $4.6 \times 10^{-6}$ & $4 \times 10^{-7}$ & Dipole field \\
\hline$\rho\left(\mathrm{amu} \mathrm{m}^{-3}\right)$ & $10^{8}$ & $4 \times 10^{10}$ & $\begin{array}{l}\text { Ionosphere: see }{ }^{\text {a }} \text {; torus: } \\
\text { Kivelson et al. }[2004]\end{array}$ \\
\hline$v_{i n}\left(\mathrm{~s}^{-1}\right)$ & 3 & 0 & See ${ }^{c}$; torus: no collision \\
\hline$v_{e n}\left(\mathrm{~s}^{-1}\right)$ & 0 & 0 & See ${ }^{d}$ \\
\hline$\kappa\left(\mathrm{kg} \mathrm{s}^{-1} \mathrm{~m}^{-3}\right)$ & 0 & $1.4 \times 10^{-23}$ & $\begin{array}{c}1000 \mathrm{~kg} / \mathrm{s} \text {, torus at } 10 \mathrm{R}_{\mathrm{J}} \\
\text { with a radius of } 1 \mathrm{R}_{\mathrm{J}}\end{array}$ \\
\hline
\end{tabular}

${ }^{a}$ The typical mass density and typical pressure of the ionosphere are difficult to evaluate since our inner boundary is located at $4.5 \mathrm{R}_{\mathrm{J}}$. A typical mass density of $10^{8}$ amu $\mathrm{m}^{-3}$ is chosen and a typical pressure of $2 \times 10^{-9} \mathrm{~Pa}$ for the electrons and for the ions.

${ }^{b}$ We assume that a current of 100 MA [cf. Khurana et al., 2004] is flowing through the ionosphere in a small region located between $13^{\circ}$ and $19^{\circ}$ latitude (the auroral region).

${ }^{\mathrm{c}}$ To achieve a conductance of $1 \mathrm{~S}$

${ }^{\mathrm{d}}$ Since we do not have electrons in the code; which is not an issue as $v_{i n}$ is chosen to obtain standard values for the Pedersen conductance (section 3.1).

Table A2. Typical Timescales for Each Term of the Continuity Equation

\begin{tabular}{lcc}
\hline & Ionosphere & Torus \\
\hline$\frac{\partial \rho}{\partial t}$ & $1.3 \times 10^{3} \mathrm{~s}$ & $5.6 \times 10^{2} \mathrm{~s}$ \\
$\nabla \cdot(\rho \mathbf{v})$ & $1.3 \times 10^{3} \mathrm{~s}$ & $5.6 \times 10^{2} \mathrm{~s}$ \\
$-\kappa$ & - & $4.7 \times 10^{6} \mathrm{~s}$ \\
\hline
\end{tabular}

Table A3. Typical Timescales for Each Term of the Momentum Equation $^{\mathrm{a}}$

\begin{tabular}{lcc}
\hline & Ionosphere & Torus \\
\hline$\nabla \cdot\left(\frac{\mathbf{B B}}{\mu_{0}}\right)$ & $4 \times 10^{-2} \mathrm{~s}$ & $4.7 \times 10^{3} \mathrm{~s}$ \\
$-\nabla \cdot\left(\underline{\underline{\mathbf{I}}} \frac{B^{2}}{2 \mu_{0}}\right)$ & $8 \times 10^{-2} \mathrm{~s}$ & $9.5 \times 10^{3} \mathrm{~s}$ \\
$-\rho_{i} v_{i n} \mathbf{v}_{\mathbf{n}}$ & $3.3 \times 10^{-1} \mathrm{~s}$ & - \\
$\nu_{i n} \rho \mathbf{v}$ & $3.3 \times 10^{-1} \mathrm{~s}$ & - \\
$-\nabla \cdot(\underline{\mathbf{I}} p)$ & $1.7 \times 10^{2} \mathrm{~s}$ & $1.8 \times 10^{4} \mathrm{~s}$ \\
$-\rho \mathbf{g}$ & $8.5 \times 10^{2} \mathrm{~s}$ & $9.3 \times 10^{3} \mathrm{~s}$ \\
$\frac{\partial}{\partial t}(\rho \mathbf{v})$ & $1.3 \times 10^{3} \mathrm{~s}$ & $5.6 \times 10^{2} \mathrm{~s}$ \\
$\nabla \cdot(\rho \mathbf{v v})$ & $1.3 \times 10^{3} \mathrm{~s}$ & $5.6 \times 10^{2} \mathrm{~s}$ \\
$-\kappa \mathbf{v}_{\mathbf{n}}$ & - & $6.1 \times 10^{6} \mathrm{~s}$ \\
\hline$\frac{m_{e}}{e} v_{i n} \mathbf{j}$ & $5.5 \times 10^{5} \mathrm{~s}$ & - \\
$\nabla \cdot\left(\frac{m_{e}}{n e^{2}} \mathbf{j} \mathbf{j}\right)$ & $1.9 \times 10^{12} \mathrm{~s}$ & $3.1 \times 10^{18} \mathrm{~s}$ \\
\hline
\end{tabular}

${ }^{\text {a }}$ The terms below the gray line are neglected since their typical timescales are very large.
Table A4. Typical Timescales for Each Term of the Induction Equation $^{\mathrm{a}}$

\begin{tabular}{llc}
\hline & Ionosphere & Torus \\
\hline$\frac{\partial \mathbf{B}}{\partial t}$ & $1.3 \times 10^{3} \mathrm{~s}$ & $5.6 \times 10^{2} \mathrm{~s}$ \\
$-\nabla \times(\mathbf{v} \times \mathbf{B})$ & $1.3 \times 10^{3} \mathrm{~s}$ & $5.6 \times 10^{2} \mathrm{~s}$ \\
$\nabla \times\left(\frac{1}{n e}(\mathbf{j} \times \mathbf{B})\right)$ & $1.1 \times 10^{6} \mathrm{~s}$ & $1.9 \times 10^{7} \mathrm{~s}$ \\
\hline$-\nabla \times\left(\frac{1}{n e} \nabla p_{e}\right)$ & $1.9 \times 10^{8} \mathrm{~s}$ & $2.5 \times 10^{8} \mathrm{~s}$ \\
$\nabla \times\left(\frac{m_{e}}{e}\left(v_{i n}\right)\left(\mathbf{v}-\mathbf{v}_{\mathbf{n}}\right)\right)$ & $3.4 \times 10^{8} \mathrm{~s}$ & - \\
$\nabla \times\left(\frac{m_{e}}{e n m_{i}} \nabla p_{i}\right)$ & $3.5 \times 10^{11} \mathrm{~s}$ & $3.4 \times 10^{10} \mathrm{~s}$ \\
$\nabla \times\left(\frac{m_{e}^{2}}{e^{2} n m_{i}}\left(v_{i n}\right) \mathbf{j}\right)$ & $5.5 \times 10^{14} \mathrm{~s}$ & - \\
$\nabla \times\left(\frac{m_{e}}{n e^{2}} \frac{\partial \mathbf{j}}{\partial t}\right)$ & $1.2 \times 10^{15} \mathrm{~s}$ & $7.4 \times 10^{14} \mathrm{~s}$ \\
$\nabla \times\left(\frac{m_{e}}{n e^{2}} \nabla \cdot(\mathbf{v j})\right)$ & $1.2 \times 10^{15} \mathrm{~s}$ & $7.4 \times 10^{14} \mathrm{~s}$ \\
$\nabla \times\left(\frac{m_{e}}{n e^{2}} \nabla \cdot(\mathbf{j v})\right)$ & $1.2 \times 10^{15} \mathrm{~s}$ & $7.4 \times 10^{14} \mathrm{~s}$ \\
$-\nabla \times\left(\frac{m_{e}}{n e^{2}} \nabla \cdot\left(\frac{1}{e n} \mathbf{j} \mathbf{j}\right)\right)$ & $1.1 \times 10^{18} \mathrm{~s}$ & $2.5 \times 10^{19} \mathrm{~s}$ \\
\hline
\end{tabular}

${ }^{\text {a }}$ The terms below the gray line are neglected since their typical timescales are very large.

given in Tables A2 to A5: One can retrieve them by equating all the terms of the first columns to zero in these tables. The associated timescales for the continuity (Table A2), momentum (Table A3), induction (Table A4), and energy (Table A5) equations are shown as well in the second and third columns. These timescales can be used to evaluate the importance of each term in the ionosphere and in the Io torus for our model. In these tables, the neglected terms are separated from the other terms by a horizontal gray line.

\section{Appendix B: Implications of $B_{r}=0$ on a Sphere With Radius $R$}

[55] Here we show that a vanishing radial component of the magnetic field $B_{r}=0$ on a sphere with radius $r=R$ implies

$$
\oint \mathrm{d} \mathbf{s} \cdot \mathbf{j}_{\perp}=0
$$

on any closed loop on the sphere. In this expression, $\mathbf{j}_{\perp}$ denotes the electric current perpendicular to the radial direction, i.e., $\mathbf{j}_{\perp}$ lies on the sphere. This statement has direct consequences for the field topology above the planet's ionosphere and in the magnetosphere as discussed in section 2.1.

[56] Derivation: The magnetic field $\mathbf{B}$ is a solenoidal vector field which thus can be written as the sum of a poloidal field [e.g., Chandrasekhar, 1961; Stern, 1976]

$$
\mathbf{P}(p)=\nabla \times \nabla \times(p \mathbf{r})
$$

and a toroidal field

$$
\mathbf{T}(p)=\nabla \times(t \mathbf{r})
$$

such that

$$
\mathbf{B}=\mathbf{P}(p)+\mathbf{T}(t),
$$

where $p(r, \theta, \phi)$ and $t(r, \theta, \phi)$ are the poloidal and toroidal potential fields, $\mathbf{r}$ is a vector in the radial direction, and $\theta, \phi$ are colatitude and azimuth in spherical coordinates. 
Table A5. Typical Timescales for Each Term of the Total Energy Equation $^{\mathrm{a}}$

\begin{tabular}{|c|c|c|}
\hline & Ionosphere & Torus \\
\hline$-\nabla \cdot\left(\mathbf{v} \cdot \frac{\mathbf{B B}}{\mu_{0}}\right)$ & $6.5 \times 10^{2} \mathrm{~s}$ & $2.9 \times 10^{3} \mathrm{~s}$ \\
\hline$\frac{\partial U}{\partial t}$ & $1.3 \times 10^{3} \mathrm{~s}$ & $5.6 \times 10^{2} \mathrm{~s}$ \\
\hline$\nabla \cdot(U \mathbf{v})$ & $1.3 \times 10^{3} \mathrm{~s}$ & $5.6 \times 10^{2} \mathrm{~s}$ \\
\hline$\nabla \cdot\left(\frac{B^{2}}{2 \mu_{0}} \mathbf{v}\right)$ & $1.3 \times 10^{3} \mathrm{~s}$ & $5.7 \times 10^{3} \mathrm{~s}$ \\
\hline$-\frac{3 v_{i n}}{\chi+1}\left(\frac{\rho}{\rho_{n}} p_{n}-p_{i}\right)$ & $1.4 \times 10^{3} \mathrm{~s}$ & - \\
\hline$-v_{n}^{2} \rho \frac{v_{m}}{\chi+1}$ & $7.5 \times 10^{3} \mathrm{~s}$ & - \\
\hline$-\mathbf{v}_{\mathbf{n}} \cdot \mathbf{v} \rho\left(v_{i n} \frac{\chi-1}{\chi+1}\right)$ & $1.6 \times 10^{4} \mathrm{~s}$ & - \\
\hline$v^{2} \rho \frac{\chi}{\chi+1} v_{i n}$ & $1.6 \times 10^{4} \mathrm{~s}$ & - \\
\hline$\frac{\mathbf{B}}{\mu_{0}} \cdot \nabla \times\left(\frac{1}{n e}(\mathbf{j} \times \mathbf{B})\right)$ & $5.5 \times 10^{5} \mathrm{~s}$ & $9.7 \times 10^{7} \mathrm{~s}$ \\
\hline$\nabla \cdot(p \mathbf{v})$ & $2.7 \times 10^{6} \mathrm{~s}$ & $1.1 \times 10^{4} \mathrm{~s}$ \\
\hline$-\rho \mathbf{v} \cdot \mathbf{g}$ & $1.3 \times 10^{7} \mathrm{~s}$ & $5.7 \times 10^{3} \mathrm{~s}$ \\
\hline$-\frac{1}{2} \kappa v_{n}^{2}$ & - & $9.5 \times 10^{6} \mathrm{~s}$ \\
\hline$-\frac{3 \kappa p_{n}}{\chi \rho_{n}}$ & - & $7 \times 10^{7} \mathrm{~s}$ \\
\hline$-\frac{\mathbf{B}}{\mu_{0}} \cdot \nabla \times\left(\frac{1}{n e} \nabla p_{e}\right)$ & $9.5 \times 10^{7} \mathrm{~s}$ & $8.2 \times 10^{8} \mathrm{~s}$ \\
\hline$\frac{\mathbf{B}}{\mu_{0}} \cdot \nabla \times\left(\frac{m_{e}}{e} \nu_{i n} \mathbf{v}\right)$ & $1.7 \times 10^{8} \mathrm{~s}$ & - \\
\hline$-\frac{\mathbf{B}}{\mu_{0}} \cdot \nabla \times\left(\frac{m_{e}}{e} \mathcal{V}_{i n} \mathbf{v}_{\mathbf{n}}\right)$ & $1.7 \times 10^{8} \mathrm{~s}$ & - \\
\hline$-\frac{5}{2} \frac{p_{e} m_{i}}{e}\left(\frac{1}{\rho} \nabla \cdot \mathbf{j}+\mathbf{j} \cdot \nabla \frac{1}{\rho}\right)$ & $9.5 \times 10^{8} \mathrm{~s}$ & $4.5 \times 10^{10} \mathrm{~s}$ \\
\hline$-\frac{3}{2} \frac{1}{\rho e} \mathbf{j} \cdot \nabla\left(m_{i} p_{e}\right)$ & $3.2 \times 10^{9} \mathrm{~s}$ & $7.5 \times 10^{10} \mathrm{~s}$ \\
\hline $\mathbf{j} \cdot \mathbf{v}_{\mathbf{n}} \frac{2 m_{n} n}{e \rho} v_{i n} \frac{m_{c} m_{i}}{m_{i}+m_{n}}$ & $6.5 \times 10^{9} \mathrm{~s}$ & - \\
\hline$-\mathbf{j} \cdot \mathbf{v} v_{i n} \frac{m_{e}\left(m_{n}-m_{i}\right)}{e\left(m_{i}+m_{n}\right)}$ & $2.6 \times 10^{10} \mathrm{~s}$ & - \\
\hline$\frac{\mathbf{B}}{\mu_{0}} \cdot \nabla \times\left(\frac{m_{e}}{e n m_{i}} \nabla p_{i}\right)$ & $3.4 \times 10^{11} \mathrm{~s}$ & $1.1 \times 10^{11} \mathrm{~s}$ \\
\hline$\frac{5}{2} \frac{p_{i} m_{e}}{e}\left(\frac{1}{\rho} \nabla \cdot \mathbf{j}+\mathbf{j} \cdot \nabla \frac{1}{\rho}\right)$ & $1.8 \times 10^{12} \mathrm{~s}$ & $6.2 \times 10^{12} \mathrm{~s}$ \\
\hline$\frac{3}{2} \frac{1}{\rho e} \mathbf{j} \cdot \nabla\left(m_{e} p_{i}\right)$ & $6 \times 10^{12} \mathrm{~s}$ & $10^{13} \mathrm{~s}$ \\
\hline$\frac{\mathbf{B}}{\mu_{0}} \cdot \nabla \times\left(\frac{m_{e}^{2}}{e^{2} n m_{i}} v_{i n} \mathbf{j}\right)$ & $2.8 \times 10^{14} \mathrm{~s}$ & - \\
\hline$\frac{\mathbf{B}}{\mu_{0}} \cdot \nabla \times\left(\frac{m_{e}}{n e^{2}} \frac{\partial \mathbf{j}}{\partial t}\right)$ & $3 \times 10^{14} \mathrm{~s}$ & $3.8 \times 10^{15} \mathrm{~s}$ \\
\hline$\frac{\mathbf{B}}{\mu_{0}} \cdot \nabla \times\left(\frac{m_{e}}{n e^{2}} \nabla \cdot(\mathbf{v j}+\mathbf{j} \mathbf{v})\right)$ & $3 \times 10^{14} \mathrm{~s}$ & $3.8 \times 10^{15} \mathrm{~s}$ \\
\hline$-j^{2} v_{i n} \frac{m_{n} n}{e^{2} \rho^{2}} \frac{m_{e}^{2} m_{i}}{m_{i}+m_{n}}$ & $2.2 \times 10^{16} \mathrm{~s}$ & - \\
\hline $\mathbf{v} \cdot \nabla \cdot\left(\frac{m_{e}}{n e^{2}} \mathbf{j} \mathbf{j}\right)$ & $3 \times 10^{16} \mathrm{~s}$ & $1.5 \times 10^{16} \mathrm{~s}$ \\
\hline$-\frac{\mathbf{B}}{\mu_{0}} \cdot \nabla \times\left(\frac{m_{e}}{n e^{2}} \nabla \cdot\left(\frac{1}{e n} \mathbf{j} \mathbf{j}\right)\right)$ & $5.3 \times 10^{17} \mathrm{~s}$ & $1.3 \times 10^{20} \mathrm{~s}$ \\
\hline$-\frac{m_{i} m_{e} \kappa}{\rho^{2} e^{2}} j^{2}$ & - & $2.9 \times 10^{21} \mathrm{~s}$ \\
\hline
\end{tabular}

${ }^{a}$ The terms below the gray line are neglected since their typical timescales are very large.

[57] The conditions $B_{r}=P_{r}+T_{r}=0$ implies $P_{r}=0$ since toroidal fields have no radial component, i.e., $T_{r}=0$. The radial component of the poloidal field can be written as [Chandrasekhar, 1961, equation (6), appendix III]

$$
P_{r}=-\frac{1}{r} L^{2}[p]=0
$$

with

$$
L^{2}=\frac{1}{\sin \theta} \frac{\partial}{\partial \theta}\left(\sin \theta \frac{\partial}{\partial \theta}\right)+\frac{1}{\sin ^{2} \theta} \frac{\partial^{2}}{\partial \phi^{2}}
$$

being the angular part of the Laplace operator. If $p$ is regular, i.e., if it has no singularity, then equation (B5) implies

$$
p=p(r) .
$$

[58] The electric current density $\mathbf{j}$ is given by

$$
\begin{gathered}
\mu_{0} \mathbf{j}=\nabla \times \mathbf{B}=\nabla \times \mathbf{T}(t)+\nabla \times \mathbf{P}(p) \\
=\mathbf{P}(t)+\mathbf{T}(-\Delta p(r)) \\
=\mathbf{P}(t)+\mathbf{T}(f(r))
\end{gathered}
$$

where we use that the curl of a poloidal field is a toroidal field and vice versa [e.g., Chandrasekhar, 1961, equations (9) and (10), appendix III]. The function $f$ depends on $r$ only. Let us consider the $\perp$-component of (B10), i.e., the component perpendicular to the radial direction

$$
\mu_{0} \mathbf{j}_{\perp}=\left.\mathbf{P}(t)\right|_{\perp}+\left.\mathbf{T}(f(r))\right|_{\perp} .
$$

The second term of the RHS in (B11) is zero since $T_{\phi}\left(t^{\prime}\right)=$ $-\partial_{\theta} t^{\prime}$ and $T_{\theta}\left(t^{\prime}\right)=(1 / \sin \theta) \partial_{\phi} t^{\prime}$ [e.g., Chandrasekhar, 1961, equation (5), appendix III]. Using equation (27b) in Stern [1976], the poloidal part in (B11) can be rewritten as

$$
\mu_{0} \mathbf{j}_{\perp}=\left.\nabla\left(\frac{\partial}{\partial r}(t \mathbf{r})\right)\right|_{\perp}-\left.\mathbf{r} \Delta t\right|_{\perp}
$$

where the last term is zero. Integration of (B12) over any closed loop on the sphere with radius $r=R$ and using Stokes' theorem thus leads to

$$
\oint \mathrm{d} \mathbf{s} \cdot \mathbf{j}_{\perp}=0
$$

Therefore, the averaged azimuthal component of the electric current over a closed loop along any fixed latitude vanishes, i.e.,

$$
\int_{0}^{2 \pi} \mathrm{d} \phi j_{\phi} \equiv<j_{\phi}>=0
$$

[59] Acknowledgments. We are grateful to F. M. Neubauer for valuable discussions and comments; and to R. Keppens and Z. Meliani for the help provided with AMRVAC. We also thank J. Raeder for helpful discussions. These results were obtained within Schwerpunktprogramm "Planetary Magnetism" of die Deutsche Forschungsgemeinschaft under grant SA 1772/1-2 and within the framework of the following projects: GOA/2009-009 (KU Leuven), G.0729.11 (FWO-Vlaanderen), and C 90347 (ESA Prodex 9). Financial support by the European Commission through the SPACECAST (project $\mathrm{n} 262468$, fp7-spacecast.eu) and eHeroes (project n 284461, www.eheroes.eu) projects is also gratefully acknowledged. The numerical results were obtained on the following HPC clusters: VIC3 of the KU Leuven, Cheops of the Universität zu Köln, and HLRB II of the Leibniz-Rechenzentrum.

[60] Masaki Fujimoto thanks the reviewers for their assistance in evaluating this paper.

\section{References}

Bagenal, F., and P. A. Delamere (2011), Flow of mass and energy in the magnetospheres of Jupiter and Saturn, J. Geophys. Res., 116 A05209, doi:10.1029/2010JA016294.

Ballester, G. E., M. A. McGrath, D. F. Strobel, X. Zhu, P. D. Feldman, and $\mathrm{H}$. W. Moos (1994), Detection of the $\mathrm{SO}_{2}$ atmosphere on Io with the Hubble Space Telescope, Icarus, 111, 2-17, doi:10.1006/icar. 1994.1129.

Bunce, E. J., and S. W. H. Cowley (2001), Divergence of the equatorial current in the dawn sector of Jupiter's magnetosphere: Analysis of Pioneer and Voyager magnetic field data, Planet. Space Sci., 49, 1089-1113, doi:10.1016/S0032-0633(01)00004-6.

Chandrasekhar, S. (1961), Hydrodynamic and hydromagnetic stability, in International Series of Monographs on Physics, 622 p., Clarendon, Oxford, U.K. 
Chané, E., S. Poedts, and B. van der Holst (2008), On the combination of ACE data with numerical simulations to determine the initial characteristics of a CME, Astron. Astrophys., 492, L29-L32, doi:10.1051/00046361:200811022.

Clarke, J. T., D. Grodent, S. W. H. Cowley, E. J. Bunce, P. Zarka, J. E. P. Connerney, and T. Satoh (2004), Jupiter's aurora, in Jupiter. The Planet, Satellites and Magnetosphere, edited by F. Bagenal, T. E. Dowling, and W. B. McKinnon, pp. 639-670, Cambridge Univ. Press, Cambridge, U.K.

Cowley, S. W. H., and E. J. Bunce (2001), Origin of the main auroral oval in Jupiter's coupled magnetosphere-ionosphere system, Planet. Space Sci., 49, 1067-1088.

Cowley, S. W. H., J. D. Nichols, and E. J. Bunce (2002), Distributions of current and auroral precipitation in Jupiter's middle magnetosphere computed from steady-state Hill-Pontius angular velocity profiles: Solutions for current sheet and dipole magnetic field models, Planet. Space Sci., 50, 717-734, doi:10.1016/S0032-0633(02)00046-6.

Cowley, S. W. H., E. J. Bunce, and J. D. Nichols (2003), Origins of Jupiter's main oval auroral emissions, J. Geophys. Res. A: Space Phys., 108(A4), 8002, doi:10.1029/2002JA009329.

Frank, L. A., W. R. Paterson, and K. K. Khurana (2002), Observations of thermal plasmas in Jupiter's magnetotail, J. Geophys. Res. A: Space Phys., 107, 1003, doi:10.1029/2001JA000077.

Fukazawa, K., T. Ogino, and R. J. Walker (2005), Dynamics of the Jovian magnetosphere for northward interplanetary magnetic field (IMF), Geophys. Res. Lett., 32, L03,202, doi:10.1029/2004GL021392.

Hansen, K. C., T. I. Gombosi, D. L. Dezeeuw, C. P. T. Groth, and K. G. Powell (2000), A 3D global MHD simulation of Saturn's magnetosphere, Adv. Space Res., 26, 1681-1690, doi:10.1016/S0273-1177(00)00078-8.

Hill, T. W. (1976), Interchange stability of a rapidly rotating magnetosphere, Planet. Space Sci., 24, 1151-1154, doi:10.1016/0032-0633(76)90152-5.

Hill, T. W. (1979), Inertial limit on corotation, J. Geophys. Res., 84, 6554-6558, doi:10.1029/JA084iA11p06554.

Hill, T. W. (2001), The Jovian auroral oval, J. Geophys. Res., 106, 8101-8108, doi:10.1029/2000JA000302.

Huang, T. S., and T. W. Hill (1989), Corotation lag of the Jovian atmosphere, ionosphere, and magnetosphere, J. Geophys. Res., 94, 3761-3765, doi:10.1029/JA094iA04p03761.

Ioannidis, G., and N. Brice (1971), Plasma densities in the Jovian magnetosphere: Plasma slingshot or Maxwell Demon? Icarus, 14, 360 doi:10.1016/0019-1035(71)90007-8.

Jia, X., M. G. Kivelson, and T. I. Gombosi (2012), Driving Saturn's magnetospheric periodicities from the upper atmosphere/ionosphere, $J$. Geophys. Res. Space Phys., 117, A04215, doi:10.1029/2011JA017367.

Joy, S. P., M. G. Kivelson, R. J. Walker, K. K. Khurana, C. T. Russell, and T. Ogino (2002), Probabilistic models of the Jovian magnetopause and bow shock locations, J. Geophys. Res., 107(A10), 1309, doi:10.1029/2001JA009146.

Keppens, R., Z. Meliani, B. van der Holst, and F. Casse (2008), Extragalactic jets with helical magnetic fields: Relativistic MHD simulations, Astron. Astrophys., 486, 663-678, doi:10.1051/0004-6361: 20079174.

Keppens, R., Z. Meliani, A. J. van Marle, P. Delmont, A. Vlasis, and B. van der Holst (2012), Parallel, grid-adaptive approaches for relativistic hydro and magnetohydrodynamics, J. Comput. Phys., 231, 718-744, doi:10.1016/j.jcp.2011.01.020.

Khurana, K. K. (2001), Influence of solar wind on Jupiter's magnetosphere deduced from currents in the equatorial plane, J. Geophys. Res., 106, 25,999-26,016, doi:10.1029/2000JA000352.

Khurana, K. K., M. G. Kivelson, V. M. Vasyliunas, N. Krupp, J. Woch, A. Lagg, B. H. Mauk, and W. S. Kurth (2004), The configuration of Jupiter's magnetosphere, in Jupiter. The Planet, Satellites and Magnetosphere, edited by F. Bagenal, T. E. Dowling, and W. B. McKinnon, pp. 593-616, Cambridge Univ. Press, Cambridge, U.K.

Kivelson, M. G., F. Bagenal, W. S. Kurth, F. M. Neubauer, C. Paranicas, and J. Saur (2004), Magnetospheric interactions with satellites, in Jupiter. The Planet, Satellites and Magnetosphere, edited by F. Bagenal, T. E. Dowling, and W. B. McKinnon, pp. 513-536, Cambridge Univ. Press, Cambridge, U.K.

Knight, S. (1973), Parallel electric fields, Planet. Space Sci., 21, 741-750, doi:10.1016/0032-0633(73)90093-7.

Krupp, N., et al. (2004), Dynamics of the Jovian magnetosphere, in Jupiter. The Planet, Satellites and Magnetosphere, edited by F. Bagenal, T. E. Dowling, and W. B. McKinnon, pp. 617-638, Cambridge Univ. Press, Cambridge, U.K.

McNutt, R. L., J. W. Belcher, and H. S. Bridge (1981), Positive ion observations in the middle magnetosphere of Jupiter, J. Geophys. Res., 86, 8319-8342, doi:10.1029/JA086iA10p08319.

Meliani, Z., R. Keppens, and B. Giacomazzo (2008), Faranoff-Riley type I jet deceleration at density discontinuities. Relativistic hydrodynam- ics with a realistic equation of state, Astron. Astrophys., 491, 321-337, doi:10.1051/0004-6361:20079185.

Miyoshi, T., and K. Kusano (1997), MHD simulation of a rapidly rotating magnetosphere interacting with the external plasma flow, Geophys. Res. Lett., 24, 2627-2630, doi:10.1029/97GL52739.

Moriguchi, T., A. Nakamizo, T. Tanaka, T. Obara, and H. Shimazu (2008), Current systems in the Jovian magnetosphere, J. Geophys. Res., 113, A05,204, doi:10.1029/2007JA012751.

Nichols, J., and S. Cowley (2004), Magnetosphere-ionosphere coupling currents in Jupiter's middle magnetosphere: Effect of precipitationinduced enhancement of the ionospheric Pedersen conductivity, Ann. Geophys., 22, 1799-1827, doi:10.5194/angeo-22-1799-2004.

Nichols, J. D., and S. W. H. Cowley (2003), Magnetosphere-ionosphere coupling currents in Jupiter's middle magnetosphere: Dependence on the effective ionospheric Pedersen conductivity and iogenic plasma mass outflow rate, Ann. Geophys., 21, 1419-1441, doi:10.5194/angeo-211419-2003.

Ogino, T., R. J. Walker, and M. G. Kivelson (1998), A global magnetohydrodynamic simulation of the Jovian magnetosphere, J. Geophys. Res., 103, 225-235, doi:10.1029/97JA02247.

Pedersen, P. (1927), The propagation of radio waves, in Danmarks naturvidenskabelige samfund, 224 pp., Gad.

Pontius, D. H. (1997), Radial mass transport and rotational dynamics, J. Geophys. Res., 102, 7137-7150, doi:10.1029/97JA00289.

Radioti, A., J. Gérard, D. Grodent, B. Bonfond, N. Krupp, and J. Woch (2008), Discontinuity in Jupiter's main auroral oval, J. Geophys. Res., 113, A01,215, doi:10.1029/2007JA012610.

Ray, L. C., Y.-J. Su, R. E. Ergun, P. A. Delamere, and F. Bagenal (2009), Current-voltage relation of a centrifugally confined plasma, J. Geophys. Res., 114, A04214, doi:10.1029/2008JA013969.

Ray, L. C., R. E. Ergun, P. A. Delamere, and F. Bagenal (2010), Magnetosphere-ionosphere coupling at Jupiter: Effect of field-aligned potentials on angular momentum transport, J. Geophys. Res., 115, A09211, doi:10.1029/2010JA015423.

Ray, L. C., R. E. Ergun, P. A. Delamere, and F. Bagenal (2012), Magnetosphere-ionosphere coupling at Jupiter: A parameter space study, J. Geophys. Res., 117, A01205, doi:10.1029/2011JA016899.

Saur, J. (2004), Turbulent heating of Jupiter's middle magnetosphere, Astrophys. J., 602, L137-L140, doi:10.1086/382588.

Saur, J., B. H. Mauk, A. Kaßner, and F. M. Neubauer (2004a), A model for the azimuthal plasma velocity in Saturn's magnetosphere, J. Geophys. Res. Space Phys., 109, A05,217, doi:10.1029/2003JA010207.

Saur, J., F. M. Neubauer, J. E. P. Connerney, P. Zarka, and M. G. Kivelson (2004b), Plasma interaction of Io with its plasma torus, in Jupiter. The Planet, Satellites and Magnetosphere, edited by F. Bagenal, T. E. Dowling, and W. B. McKinnon, pp. 537-560, Cambridge Univ. Press, Cambridge, U.K

Schunk, R. W. (1975), Transport equations for aeronomy, Planet. Space Sci. 23, 437-485, doi:10.1016/0032-0633(75)90118-X.

Schure, K. M., J. Vink, A. Achterberg, and R. Keppens (2009), Evolution of magnetic fields and cosmic ray acceleration in supernova remnants, $A d v$. Space Res., 44, 433-439, doi:10.1016/j.asr.2009.05.010.

Stern, D. P. (1976), Representation of magnetic fields in space, Rev Geophys. Space Phys., 14, 199-214.

Strobel, D. F., and S. K. Atreya (1983), Ionosphere, in Physics of the Jovian Magnetosphere, edited by A. J. Dessler, pp. 51-67, Cambridge Univ. Press, New York.

Tanaka, T. (1994), Finite volume TVD scheme on an unstructured grid system for three-dimensional MHD simulation of inhomogeneous systems including strong background potential fields, J. Comput. Phys., 111, 381-390, doi:10.1006/jcph.1994.1071.

Tao, C., H. Fujiwara, and Y. Kasaba (2010), Jovian magnetosphereionosphere current system characterized by diurnal variation of ionospheric conductance, Planet. Space Sci., 58, 351-364, doi:10.1016/ j.pss.2009.10.005.

Thomas, N., F. Bagenal, T. W. Hill, and J. K. Wilson (2004), The Io neutral clouds and plasma torus, in Jupiter. The Planet, Satellites and Magnetosphere, edited by F. Bagenal, T. E. Dowling, and W. B. McKinnon, pp. 561-591, Cambridge Univ. Press, Cambridge, U.K

Tóth, G. (1996), A general code for modeling MHD flows on parallel computers: Versatile advection code, Astrophys. Lett. Commun., 34, 245-250.

van der Holst, B., and R. Keppens (2007), Hybrid block-AMR in cartesian and curvilinear coordinates: MHD applications, J. Comput. Phys., 226, 925-946, doi:10.1016/j.jcp.2007.05.007.

van der Holst, B., C. Jacobs, and S. Poedts (2007), Simulation of a breakout coronal mass ejection in the solar wind, Astrophys. J., 671, L77-L80, doi: $10.1086 / 524732$. 


\section{CHANÉ ET AL.: JUPITER'S MAGNETOSPHERE}

Vasyliunas, V. M. (1983), Plasma distribution and flow, in Physics of the Jovian Magnetosphere, edited by A. J. Dessler, pp. 395-453, Cambridge Univ. Press, New York.

Walker, R. J., and T. Ogino (2003), A simulation study of currents in the Jovian magnetosphere, Planet. Space Sci., 51, 295-307.

Walker, R. J., T. Ogino, and M. G. Kivelson (2001), Magnetohydrodynamic simulations of the effects of the solar wind on the Jovian magnetosphere, Planet. Space Sci., 49, 237-245.
Woch, J., N. Krupp, A. Lagg, and A. Tomás (2004), The structure and dynamics of the Jovian energetic particle distribution, Adv. Space Res., 33, 2030-2038, doi:10.1016/j.asr.2003.04.050.

Yelle, R. V., and S. Miller (2004), Jupiter's thermosphere and ionosphere, in Jupiter. The Planet, Satellites and Magnetosphere, edited by F. Bagenal, T. E. Dowling, and W. B. McKinnon, pp. 185-218, Cambridge Univ. Press, Cambridge, U.K 